Publ. RIMS, Kyoto Univ.

14 (1.978), 1-52

\title{
Closedness of the Douady Spaces of Compact Kähler Spaces ${ }^{1)}$
}

By

\author{
Akira FuJIK1*
}

\section{Introduction}

Let $X$ be a complex space and $D_{X}$ the Douady space of compact analytic subspaces of $X$. For every point $d \in D_{X}$, we denote by $Z_{d}$ the corresponding analytic subspace of $X$. Define the subspace, $\bar{D}_{X}$, of $D_{X \text {.red }}$ by $\bar{D}_{X}=\cup_{\alpha} D_{\alpha}$, where $D_{\alpha}$ are the irreducible components of $D_{X, \text { red }}$ such that for some $d \in D_{c} Z_{d}$ is reduced and pure dimensional. Then the main purpose of this paper is to show the following: If $X$ is a compact Kähler space, then every connected component of $\bar{D}_{x}$ is compact (Theorem 4.5). Further if $X$ is a compact complex space whose reduction $X_{\text {red }}$ is a meromorphic image of a compact Kähler space, then every irreducible component of $D_{X, \text { red }}$ is compact (Theorem 5.3).

In algebraic geometry the corresponding results are wellknown. In fact, if $X$ is a projective analytic space i.e. the one embedded in some projective space (or more generally a meromorphic image of such and hence a Moisezon space), then $D_{X}$ is a disjoint union of projective analytic spaces (resp. compact Moisezon spaces) (cf. [1] and [10]). In particular every connected component of $D_{X}$ is compact in these special cases. Note that the problem in this generality still remains open in the general case, though in most applications the results obtained here would be sufficient.

Now for the proof, instead of $D_{X}$ itself, we first fix our eyes on the 'Chow variety' of $\mathrm{X}$ whose existence in the analytic category has recently been established by Barlet [3]. We denote this space by $B(X)$. Then our proof is roughly divided into two steps: 1) we show the compactness

Received January 28, 1977. Revised August 22, 1977.

* Research Institute for Mathematical Sciences, Kyoto University, Kyoto 606, Japan.

1) Research partially supported by the Sonderforschungsbereich at the University of Bonn. 
of the connected components of $B(X)$ when $X$ is compact and Kähler, ${ }^{2)}$ and then 2) using the generic flatness theorem for proper maps due to Firsch [6] and Hironaka's flattening theorem [14] we reduce the problem to the case of $B(X)$ via the natural map $\bar{\rho}: \bar{D}_{X} \rightarrow B(X)$. Let me explain the step 1) more in detail. First note that every point of $B(X)$ corresponds to an effective compact $q$-cycle on $X$ for some $q \geqq 0$, which in turn may be considered as a current of dimension $2 q$ on $X$. Then we show that a) the natural topology of $B(X)$ is equivalent to the one induced by the weak topology on the space of currents on $X$ (Prop. 2.3). Combining this result with a result of Harvey and Shiffman in [12] on the closedness of holomorphic cycles we show that $b$ ) every bounded set on $B(X)$ is relatively compact (Prop. 2.10). Finally we show that c) if $X$ is Kähler, then every connected component of $B(X)$ is bounded (Prop. 4.1). Then 1) follows immediately from b) and c). Note that in the course of our proof c) is the only point at which we need the Kählerian assumption.

In the subsequent paper [8] we shall give an application of the results of this paper to the automorphism groups of compact Kähler manifolds, which was the original motivation for this investigation.

We now give a brief explanation on the organization of this paper. After some preliminaries on $C^{\infty}$-forms and currents etc. in $\S 1$ we introduce in $\S 2$ the space $B(X)$ according to Barlet [3] and prove a) and b) stated above. In $\S 3$ we reduce the case of $\bar{D}_{X}$ to that of $B(X)$ via the map $\bar{\rho}$ mentioned above. Here we have to treat also the relative case as it is needed in the reductions in $\S 5$. Next in $\S 4$ we prove c) together with a generalization to compact complex spaces which are meromorphic images of compact Kähler spaces. In the final section we prove our theorems in its full generality, which involves the relative Douady space, $D_{X / S}(\mathcal{E})$, of the quotient analytic sheaves of a coherent analytic sheaf $\mathcal{E}$ on $X$ with $X$ a complex space over $S$, by a series of reductions to the case of $\bar{D}_{X}$.

Conventions. In this paper all the complex spaces have a countable base for the open sets and hence paracompact. Complex spaces are not

2) This has also been shown by D. Lieberman independently. 
necessarily reduced unless otherwise stated. Let $X=\left(X, \mathcal{O}_{Y}\right)$ be a complex space. Then $X_{\text {red }}$ denotes the underlying reduced subspace, the reduction, of $X$. Usually a complex analytic subspace of $X$ is simply called a subspace of $X$. An analytic subset (resp. a subvariety) of $X$ is by definition a reduced (resp. and irreducible) analytic subspace of $X$. An open subset of $X$ is called Zariski open if it is the complement of an analytic subset of $X$. Let $S$ be a complex space and $X$ a complex space over $S$ i.e. there is a fixed morphism $f: X \rightarrow S$. Then if $T$ is another complex space over $S$ we write $X_{T}=X \times{ }_{S} T$ and $f_{T}: X_{T} \rightarrow T$ for the natural projection. Similarly if $\mathcal{E}$ is a coherent analytic sheaf on $X$, we denote by $\varepsilon_{T}$ the pullback of $\varepsilon$ on $X_{T}$ by the natural projection $X_{T} \rightarrow X$. If $T=\{s\}$ is a point we write $X_{s}\left(\right.$ resp. $\left.\mathcal{E}_{s}\right)$ instead of $X_{\{s\}}$ (resp. $\mathcal{E}_{\{s\}}$ ).

\section{§ 1 . Prelliminaroies}

1. 1. Let $X$ be a complex space. We shall define on $X$ the sheaf $\mathscr{A}_{X}^{m}$ (resp. $A_{X}^{p, q}$ ) of germs of $C^{\infty} m$-forms (resp. $C^{\infty}$ forms of type $(p, q)$ ) with direct sum decomposition $*) \mathscr{A}_{X}^{m}=\bigoplus_{p+q=m} \mathcal{A}_{X}^{p, q}$ and the differentials $d: \mathscr{A}_{X}^{m} \rightarrow \mathscr{A}_{X}^{m+1}$ (resp. $\partial: \mathscr{A}_{X}^{p, q} \rightarrow \mathcal{A}_{X}^{p+1, q}$ and $\bar{\partial} \mathscr{A}_{X}^{p, q} \rightarrow \mathcal{A}_{X}^{p, q+1}$ ) with $d=\partial+\bar{\partial}$.

First we consider the case where $X$ is a subspace of a domain $V$ in $\mathbb{C}^{n}=\mathbb{C}^{n}\left(z_{1}, \cdots, z_{n}\right)$ with the ideal sheaf $\mathcal{I}=\mathcal{I}_{X}$. Then we define $\mathscr{A}_{X}=\mathscr{A}_{x}^{0}$ by $\mathscr{A}_{X}=\mathscr{A}_{V} /(\mathcal{G}+\overline{\mathcal{G}}) \mathscr{A}_{V}$, where $\overline{\mathfrak{I}}=\{\bar{f} ; f \in \mathscr{I}\}, \bar{f}$ being the complex conjugate of $f$. Next define the $\mathscr{A}_{V}$-submodule, $\mathscr{A}_{\boldsymbol{X}}^{\prime}$, of $\mathscr{A}_{V}^{1}$ by $\mathscr{A}_{X}^{\prime}=\sum \mathcal{G} \mathcal{A} d z_{\alpha}+\sum \overline{\mathcal{G}} \mathcal{A} d z_{\beta}+\mathcal{A} d \mathcal{G}+\mathcal{A} d \overline{\mathcal{G}}, \quad \mathcal{A}=\mathcal{A}_{V}$, where $\mathcal{A} d \mathcal{G}$ $=\left\{\sum h_{r} d g_{r}, h_{r} \in \mathcal{A}\right.$ and $\left.g_{r} \in \mathcal{G}\right\}$ and similarly for $\mathcal{A} d \overline{\mathscr{J}}$. Then put $\mathcal{A}_{X}^{m}$ $=\mathscr{A}_{V}^{m} / \mathscr{A}_{X}^{\prime} \wedge \mathscr{A}_{V}^{m-1}, m \geqq 1$. These form naturally an $\mathscr{A}_{X}$-graded algebra $\mathscr{A}_{X}$. Further define the $\mathscr{A}_{V}$-submodules, $\mathscr{A}_{X}^{p, q}, p+q=m$, of $\mathscr{A}_{X}^{m}$ by $\mathcal{A}_{X, x}^{p, q}=\left\{\phi \in \mathcal{A}_{X, x}^{m} ; \exists \widetilde{\psi} \in \mathcal{A}_{V, x}^{p, q}\right.$ inducing $\left.\phi\right\}$. Then it is immediate to see that we have the direct sum decomposition *) above. Moreover the usual differentials $d$ (resp. $\partial$ and $\bar{\partial}$ ) on $\mathscr{A}_{V}^{m}$ (resp. $\mathcal{A}_{V}^{p, q}$ ) is easily seen to induce the one on $\mathcal{A}_{X}^{m}$ (resp. $\mathcal{A}_{X}^{p, q}$ ) with $d=\partial+\bar{\partial}$. On the other hand the natural complex conjugation on $\mathscr{A}_{V}^{m}$ induces a $\mathbb{C}$-antilinear involution on $\mathscr{A}_{X}^{m}$. In particular we can define the real forms on $X$ as those left fixed by this involution.

In the general case take an open covering $\mathfrak{U}=\left\{U_{\alpha}\right\}$ of $X$ with an 
embedding $j_{\alpha}: U_{\alpha} \rightarrow V_{\alpha}$ for each $\alpha$, where $V_{\alpha}$ is a domain in some $\mathbb{C}^{n_{\alpha}}$. Then we see readily that the sheaves $\left\{j_{\alpha}^{*} \mathcal{A}_{j_{\alpha}\left(U_{\alpha}\right)}^{m}\right\}$ (resp. $\left.j_{\alpha}^{*} \mathcal{A}_{j_{\alpha}\left(U_{\alpha}\right)}^{p, q}\right)$ and the differentials $\left\{j_{\alpha}^{*} d_{\alpha}\right\}$ (resp. $\left\{j_{\alpha}^{*} \partial_{\alpha}\right\}$ and $\left\{j_{\alpha}^{*} \bar{\partial}_{\alpha}\right\}$ ) naturally patch together to define the sheaves $\mathscr{A}_{X}^{m}$ (resp. $\mathcal{A}_{X}^{p, q}$ ) and the differentials $d$ (resp. $\partial$ and $\bar{\partial})$, globally defined on $X$, with natural direct some decomposition *) above and with $d=\partial+\bar{\partial}$. Similarly the notion of real forms can also be globalized. Note that even if $X$ is reduced, the above definition is a little different from that of Bloom-Herrera (cf. [15]), though both coincide with the usual definition on the nonsingular points of $X$. Quite similarly we define the space of continuous forms on $X$, and its direct sum decomposition into $(p, q)$ components as above. Next let $f: X \rightarrow Y$ be a morphism of complex spaces. Then there is a natural homomorphism $f^{*}: \mathscr{A}_{\dot{Y}} \rightarrow \mathcal{A}_{X}$ such that if $g: Y \rightarrow Z$ is another morphism, then $f^{*} g^{*}=(g f)^{*}$.

We denote by $A_{X, c}^{m}$ the space of $C^{\infty} m$-forms on $X$ with compact supports. Then the convergence in $A_{X, c}^{m}$ is defined as follows. Take a locally finite open covering $\mathfrak{l}=\left\{U_{\alpha}\right\}$ of $X$ with an embedding $j_{\alpha}: U_{\alpha} \rightarrow V_{\alpha}$ for each $\alpha$ as above. Let $\left\{\rho_{\alpha}\right\}$ be a partition of unity subordinate to the covering $\mathfrak{U}$. Taking $\mathfrak{U}$ suitably we may assume that the support of $\rho_{\alpha}$ is compact for each $\alpha$. Then we say that a sequence $\left\{\phi_{n}\right\}, \phi_{n} \in A_{\boldsymbol{X}, c}^{m}$, $n \geqq 1$, converges to $\phi_{0} \in A_{X, c}^{m}$ if the supports of $\phi_{n}$ are contained in a fixed compact $K$ and for each $\alpha$ there exists a compact set $K_{\alpha}$ in $V_{\alpha}$ and a representatives $\widetilde{\phi}_{n \alpha}$ of $\rho_{\alpha} \phi_{n}, n \geqq 0$, with support contained in $K_{\alpha}$ such that $\widetilde{\phi}_{n \alpha} \rightarrow \widetilde{\phi}_{0 \alpha}$ uniformly on $K_{\alpha}$ with all the derivatives of their coefficients. It is easy to see that this definition is independent of the choice of $\mathfrak{U}$ and $\left\{\rho_{\alpha}\right\}$ as above.

Next we define the space, $D_{X, m}^{\prime}$, of currents of dimension $m$ on $X$ as the vector space of complex valued continuous linear forms on $A_{X, c}^{m}, m \geqq 0$. The boundary operator $b: D_{X, m}^{\prime} \rightarrow D_{X, m-1}^{\prime}$ is then defined by $b(\alpha)$ $(\omega)=\alpha(d \omega)$ for all $\omega \in A_{X, c}^{m-1}$ and $\alpha \in D_{X, m}^{\prime}$. We put on $D_{X, m}^{\prime}$ the weak topology which is defined by the weak convergence of the sequences of the elements of $D_{X, m}^{\prime}$ i.e. $\alpha_{n} \in D_{X, m}^{\prime}, n \geqq 1$, converges weakly to $\alpha_{0} \in D_{X, m}^{\prime}$ if for every $\omega \in A_{X, c}^{m} \alpha_{n}(\omega)$ converges to $\alpha_{0}(\omega)$.

1. 2. We refer the definitions about semianalytic sets and their elementary properties to [13]. Let $C$ be a semianalytic set of dimension $m$ on 
a complex space $X$ with a fixed orientation on its $m$-simple points. Then Herrera in [13] showed the following: 1) (integration on semianalytic sets). For every continuous $m$-form, $\chi$, on $X$ with compact support, the integral $\int_{C} \chi$ of $\chi$ on $m$-simple points of $C$ can be defined, in such a way that it defines a current of dimension $m$ on $X$, denoted by $c[C]$. 2) (Stokes formula) we have the formula

$$
b(c[C])=c[b C],
$$

where $b C$ is the boundary of $C$ as a set which is again a semianalytic set with the natural orientation on its $(m-1)$-simple points induced from $C$. If $C$ is a complex analytic subset of $X$ with its natural orientations on the nonsingular points, then the above results are due to Lelong (cf. $[15,3.1])$. In particular $c[C]$ is then $b$-closed.

Let $q \geqq 0$ be an integer. A holomorphic $q$-cycle, or simply a $q$-cycle, is a locally finite sum, $A=\sum n_{\alpha} A_{\alpha}$, where $n_{\alpha} \in \mathbb{Z}$ and $A_{\alpha}$ are subvarieties of $X$ of dimension $q$. For a $q$-cycle $A$ we denote by $|A|$ the set $\bigcup_{\alpha} A_{\alpha}$ and call it the support of $A$. This is an analytic subset of $X$. We call $A$ effective if $n_{a} \geqq 0$ for all $\alpha . A$ is called simply a compact $q$-cycle if $A$ is effective and $|A|$ is compact. Now for every holomorphic $q$-cycle $A=\sum n_{\alpha} A_{\alpha}$ on $X$ we define a current, $c[A] \in D_{X, 2 q}^{\prime}$, by $c[A]=\sum_{\alpha} n_{\alpha} c\left[A_{\alpha}\right]$. This is welldefined, since the sum is locally finite. We often write $c[A](\chi)=\int_{A} \chi$ for every continuous $2 q$-form $\chi$ on $X$. We denote by $C_{q}^{\text {loc }}(X)$ (resp. $C_{q}(X)$ ) the set of all the currents of the form $c[A]$ with $A$ an effective (resp. compact) $q$-cycle on $X$.

We recall the notion of boundedness of a subset of $C_{q}(X)$ (cf. [15]). First for any domain $V$ in $\mathbb{C}^{n}=\mathbb{C}^{n}\left(z_{1}, \cdots, z_{n}\right)$ we call the $2 q$-form on $V$,

$$
v_{q}=(\sqrt{-1} / 2)^{q} \sum_{I} d z_{i_{1}} \wedge d \bar{z}_{i_{1}} \wedge \cdots \wedge d z_{i_{q}} \wedge d \bar{z}_{i_{q}}
$$

the summation being over all the $q$-tuples $I=\left(i_{1}, \cdots, i_{q}\right)$ with $1 \leqq i_{1}<\cdots$ $<i_{q} \leqq n$, the volume $2 q$-form associated with the standard Hermitian metric on $\mathbb{C}^{n}$. Then for every element $A \in C_{q}^{\text {loc }}(V)$ we call $\int_{A} v_{q}$ the volume of $A$ and denote it by $\operatorname{vol}(A)$.

Definition 1. H. 1) Let $V$ be a domain in $\mathbb{C}^{n}$. Then a subset $E$ $\subseteq C_{q}^{\text {loc }}(V)$ is called bounded if $\sup _{A \in E} \operatorname{vol}(A)<+\infty$. 2) Let $X$ be a com- 
plex space and $E$ a subset of $C_{q}(X)$. Then we call $E$ bounded if there exists an open covering $\mathfrak{U}=\left\{U_{\alpha}\right\}$ with an embedding $j_{\alpha}: U_{\alpha} \rightarrow V_{\alpha} \subseteq \mathbb{C}^{n_{\alpha}}$ for each $\alpha$ such that for all $\alpha$ the sets, $E_{\alpha}=\left\{c\left[j_{\alpha}\left(\left.A\right|_{U}\right)\right] ; A \in E\right\}$ are bounded in $C_{q}^{\text {loc }}\left(V_{\alpha}\right)$ in the sense of 1$)$, where $V_{\alpha}$ is a domain in $\mathbb{C}^{n_{\alpha}}$.

1.3. Let $M$ be a real connected $C^{\infty}$ manifold of dimension $2 q$ and $T_{M}^{*}$ the cotangent bundle of $M$. Suppose that $M$ is oriented and $\Omega$ any volume element on $M$. Then every real $C^{\infty} 2 q$-form $\chi$ on $M I$ can be uniquely written as $\chi=\lambda \Omega$ for a unique real $C^{\infty}$ function $\lambda$ on $M$. Then we call $\chi$ positive if $\lambda$ is positive at every point of $M$. This definition is in fact independent of the choice of the rolume element and depends only on the orientation of $M$. On the other hand, for $\gamma$ as above we write $|\chi|=|\lambda| \Omega$ with $|\lambda|$ the absolute value of $\lambda$. Then $|\chi|$ also is independent of the choice of $\Omega$. Let $Y$ be a connected complex manifold of complex dimension $q$. Then $Y$ has the natural orientation compatible with its complex structure. Thus for every real $C^{\infty} 2 q$-form on $Y$ we can speak of its positivity and of $|\chi|$.

Let $G$ be a domain in $\mathbb{C}^{n}$ and $\chi$ a real $C^{\infty}(q, q)$-form on $G$. Then we call $\chi$ positive if for every open subset $U$ of $G$ and for every connected complex submanifold $Y$ of $U$ of complex dimension $q e^{*} \chi$ is positive on $Y$, where $\iota: Y \rightarrow U$ is the inclusion. The positive forms can also be characterized by the following infinitesimal condition (cf. [23, Lemma 2.2]); $\chi$ is positive if and only if for every point $x \in G$ and every element $\psi \in \wedge^{q} T_{G, x}$ of the form $\psi=(\sqrt{-1})^{-q} e_{1} \wedge \bar{e}_{1} \cdots e_{q} \wedge \bar{e}_{q}$ with $e_{1}, \cdots, e_{q}$ linearly independent vectors in $T_{G, x}$, we have $\chi_{x}(\psi)>0$, where $T_{G}$ is the holomorphic tangent bundle of $G$ and $T_{G, x}$ is its fiber at $x$. We shall list some elementary facts on positive forms. 1) The volume $2 q$-form $v_{q}$ defined in 1.2 is positive. 2) Let $\chi$ be a positive $(1,1)$ form on $G$. Then the $q$-th exterior product $\chi^{q}, 1 \leqq q \leqq n$, is again positive. 3) If $\chi_{1}$ and $\chi_{2}$ are two positive forms on $G$, then for every relatively compact subdomain $U$ of $G$ there exist positive constants $c_{1}$ and $c_{2}$ such that $c_{1} \chi_{1}<\chi_{2}<c_{2} \chi_{2}$ i.e. $\chi_{2}-c_{1} \chi_{1}$ and $c_{2} \chi_{1}-\chi_{2}$ are positive (cf. Stoll [23, p.98]).

Definition 1.2. 1) Let $X$ be a general complex space and $\gamma$ a real $C^{\infty}(q, q)$ form on $X$. Then we call $\chi$ positive if there exists an open 
covering $\mathfrak{U}=\left\{U_{\alpha}\right\}$ of $X$ with for each $\alpha$ an embedding $j_{\alpha}: U_{\alpha} \rightarrow G_{\alpha}$ of $U_{\alpha}$ into a subdomain $G_{\alpha}$ in $\mathbb{C}^{n_{\alpha}}$ and a $C^{\infty}$ positive form, $\chi_{\alpha}$, of type $(q, q)$ on $G_{\alpha}$ such that $j_{\alpha}^{*} \chi_{\alpha}=\left.\chi\right|_{U_{\alpha}}$. 2) A $C^{\infty}$ positive $(1,1)$-form on $X$ is called a hermitian form on $X$. Further a hermitian form $\chi$ is called a Kähler form if in the above definition all the $\chi_{\alpha}$ can be taken to be closed. A complex space with a Kähler form is called a Kähler space.

Remark 1.1. 1) Using partition of unity we can always construct a Hermitian form on every complex space $X$. 2) The above definition of a Kähler space coincides with that of Moishezon in [16] because of the fact that every real closed $C^{\infty}(1,1)$ form $\chi$ on a domain $G$ is locally written as $\chi=\sqrt{-1} \partial \bar{\partial} \phi$ for some $C^{\infty}$ function $\phi$ and $\chi$ represents a Kähler form if and only if $\phi$ is a strictly plurisubharmonic function.

By virtue of 1 ) and 3 ) above on positive forms we get easily the following lemma.

Lemma 1 . 1 . Let $X$ be a complex space and $\chi$ a real positive $C^{\infty}$ form of type $(q, q)$ on $X$ with $q>0$. Let $E$ be the subset of $C_{q}(X)$ such that $\cup_{A \in E}|A|$ is contained in a compact subset of $X$. Then $E$ is bounded if and only if the set $\left\{\int_{A} \chi ; A \in E\right\}$ is bounded.

1. $\mathbb{1}_{\text {。 }}$ Let $f: X \rightarrow S$ be a proper morphism of complex spaces and $\mathcal{E}$ a coherent analytic sheaf on $X$. Suppose that $S$ is reduced. Then by Frisch [6] there exists a dense Zariski open subset $U=U(\mathcal{E}, f)$ of $S$ such that $\mathcal{E}$ is flat over $s \in S$ if and only if $s \in U$.

Let $f: X \rightarrow S$ and $\mathcal{E}$ be as above. Then a flattening of $\mathcal{E}$ is a commutative diagram

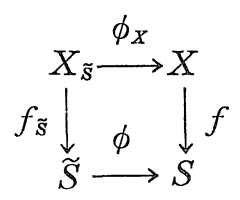

such that 1) $\phi$ is a surjective, proper and bimeromorphic morphism which gives an isomorphism of $\phi^{-1}(U)$ and $U$ with $\left.U=U(\mathcal{E}, f), 2\right) X_{\tilde{S}}=X \times{ }_{S} \widetilde{S}$, and $f_{\tilde{S}}\left(\right.$ resp. $\left.\phi_{X}\right)$ is the natural projection, and 3) $\mathcal{E}=\phi_{\mathbb{X}}^{*} \mathcal{E}$ modulo $f_{\tilde{S}^{-}}$ torsion (cf. $[14,(4.2 .1)$.$] for the definition) is flat over \widetilde{S}$. In case 
$\mathcal{E}=\mathcal{O}_{X}$ let $\widetilde{X}$ be the subspace of $X_{\widetilde{S}}$ corresponding to the quotient sheaf $\mathcal{E}=\mathcal{O}_{X}$ of $\mathcal{O}_{X_{\tilde{S}}}$, i.e. the strict transform of $X$ in $X_{\tilde{S}}$, and $\tilde{f}: \widetilde{X} \rightarrow \widetilde{S}$ the induced morphism. Then we call $\tilde{f}$ a flattening of $f$ and $\phi$ the flattening morphism. By Hironaka [14] for every $f$ and $\mathcal{E}$ as above there always exists a flattening of $\varepsilon$.

Lemma 1.2. Let $f: X \rightarrow S$ be a proper morphism of reduced complex spaces. Then there exists a Zariski open subset $U$ (resp. V) of $X$ (resp. $S$ ) such that for every $x \in U$ (resp. $\left.s \in T^{\top}\right) X_{f(x)}$ (resp. $X_{s}$ ) is nonsingular at $x$ (resp. generically reduced).

Proof. The result is more or less well-known. For the simplicity of arguments here we use the resolution by Hironaka. Let $\phi: \widetilde{S} \rightarrow S$ (resp. $\psi: \widetilde{X} \rightarrow X$ ) be a resolution of $S$ (resp. a resolution of $X_{\tilde{S} \text {, red }}$ composed with the natural projection $\left.X_{\tilde{s}, \text { red }} \rightarrow X\right)$. Let $\tilde{f}: \widetilde{X} \rightarrow \widetilde{S}$ be the induced map. Then there exists a Zariski open subset $\widetilde{V}$ of $\widetilde{S}$ such that $\tilde{f}$ is smooth at every point of $\tilde{f}^{-1}(\widetilde{V})$. Let $\widetilde{E}$ (resp. $\widetilde{F}$ ) be the nowhere dense analytic subset of $\widetilde{S}$ (resp. $\widetilde{X}$ ) such that $\phi$ (resp. () ) gives an isomorphism of $\widetilde{S}-\widetilde{E}$ and $S-\phi(\widetilde{E})$ (resp. $\widetilde{X}-\widetilde{F}$ and $\widetilde{X}-\psi(\widetilde{F}))$. Let $V=\phi(\widetilde{V} \cap(\widetilde{S}$ $-\widetilde{E}))=\phi(\widetilde{V}) \cap(S-\phi(\widetilde{E})) \quad\left(\right.$ resp. $U=\psi\left(\tilde{f}^{-1}(\widetilde{V}) \cap(\widetilde{X}-\widetilde{F})\right)=f^{-1}(V) \cap$ $(X-\psi(\widetilde{F}))$. Then it is easy to see that this $V$ (resp. U) has the desired properties.

Q.E.D.

Lemma $\mathbb{1}_{\text {.3. }}$ Let $f ; X \rightarrow S$ and $V$ be as in the previous lemma. Suppose that $X$ is pure dimensional and $S$ is irreducible. Suppose further that every irreducible component, $X_{i}$, of $X$ is mapped surjectively onto $S$. Let $\omega_{X}$ (resp. $\omega_{S}$ ) be a positive $C^{\infty}(1,1)$-form on $X$ (resp. $S$ ) and $\Omega=\omega_{X}+f^{*} \omega_{s} . \quad$ Let $n=\operatorname{dim} X, m=\operatorname{dim} S$ and $q=n-m$. Let $\lambda(v)=\int_{\left[X_{v}\right]} \omega_{X}^{q}, v \in V$, where $\left[X_{v}\right]=\sum_{\alpha} X_{\alpha v}$ with $X_{\alpha v}$ irreducible

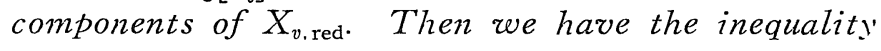

$$
\int_{X} \Omega^{n} \geqq \int_{V} \lambda(v) \omega_{S}^{m}
$$

Proof. Let $\phi: \widetilde{S} \rightarrow S, \phi: \widetilde{X} \rightarrow X$ and $\tilde{f}: \widetilde{X} \rightarrow \widetilde{S}$ be as in the proof of the previous lemma. Let $\omega_{\tilde{S}}=\phi^{*} \omega_{S}, \omega_{\tilde{X}}=\psi^{*} \omega_{Y}$ and $\widetilde{\Omega}=\omega_{\tilde{X}}+\tilde{f}^{*} \omega_{\tilde{S}}=\psi^{*} \Omega$. 
Then we have $\int_{X} \Omega^{n}=\int_{\widetilde{X}} \widetilde{\Omega}^{n}$. On the other hand, if $\widetilde{V}=\phi^{-1}(V)$ and $\tilde{\lambda}(\widetilde{\boldsymbol{V}})$ $=\int_{\left[\tilde{x}_{\tilde{v}]}\right.} \omega_{\tilde{X}}^{q}, \widetilde{v} \in \widetilde{V}$, with $\left[\widetilde{X}_{\tilde{v}}\right]$ defined as above, then $\tilde{\lambda}(\widetilde{v})=\lambda(\phi(v))$ and $\int_{V} \lambda(v) \omega_{S}^{m}=\int_{\tilde{V}} \tilde{\lambda}(\widetilde{\mathcal{v}}) \omega_{\tilde{S}}^{m}$. Hence it suffices to show the lemma when $X$ and $S$ are nonsingular and $f$ is smooth on $f^{-1}(V)$. In this case using the local coordinates we easily see that

$$
\int_{X} \Omega^{n}=\sum_{\substack{s+t=n \\ t \leqq m}} \int_{X} \omega_{X}^{s} \wedge f^{*} \omega_{S}^{t} \geqq \int \omega_{X}^{q} \wedge f^{*} \omega_{S}^{m}=\int_{V} \lambda(v) \omega_{S}^{m} .
$$

If $f$ is flat in Lemma 1.2 , then more precise result can be obtained.

Lemma 1. 1. Let $f: X \rightarrow S$ be a proper, flat and surjective morphism of complex spaces. Suppose that $X$ is pure dimensional and $S$ is reduced and irreducible. Then the following conditions are cquivalent.

1) There exists a point $s \in S$ such that $X_{s}$ is reduced.

2) The set $U=\left\{s \in S ; X_{s}\right.$ is reduced $\}$ is nonempiy and Zariski open in $S$.

3) $X$ is reduced.

Proof. The equivalence of 1) and 2) immediately follows from a result of Banica [2] which says that $U$ in 2) is Zariski open in $S$. Next we show the equivalence of 2) and 3). We put $n=\operatorname{dim} X, m$ $=\operatorname{dim} S$ and $q=n-m$. Since $f$ is flat, $X_{s}$ is pure $q$-dimensional for every $s \in S . \quad$ We put $S_{k}(X)=\left\{x \in X ; \operatorname{depth}_{x} X \leqq k\right\}$ (resp. $S_{k}(f)=\{x \in X$; $\left.\left.\operatorname{depth}_{x} X_{f(x)} \leqq k\right\}\right) \quad$ and $\quad B_{k}(X)=\left\{x ; \operatorname{dim}_{x} S_{k}(X) \geqq k\right\} \quad$ (resp. $B_{k}(f)=\{x ;$ $\left.\left.\operatorname{dim}_{x} S_{k}(f) \cap X_{f(x)} \geqq k\right\}\right)$. Let $A=\{x \in X ; X$ is not reduced at $x\}$ (resp. $A_{f}=\left\{x \in X ; X_{f(x)}\right.$ is not reduced at $\left.\left.x\right\}\right)$. Assume that $q>0$, since the result is well-known if $q=0$.

We first consider the case where $S$ is nonsingular and $X_{s}$ is generjcally reduced for every $s \in S$. Note that in this case $X$ also is generically reduced. Since $f$ is flat and $S$ is nonsingular, we get that $\operatorname{depth}_{x} X$ $=$ depth ${ }_{x} X_{s}+m$ for all $s \in S$ and $x \in X_{s}$. Hence $S_{k}(f)=S_{k}, m(X)$ for every $k \geqq 0$. On the other hand, by Houzel's criterion cited in [2] applied 
to our pure dimensional case, combined with Serre's criterion of reducedness (c.f. [2]), it follows that $A=\bigcup_{n-1 \geq k \geq m} B_{k}(X)$ and $A_{f}=\bigcup_{q-1 \geq k \geq 0} B_{k}(f)$. Suppose first that $U$ in 2) is empty so that $f\left(A_{f}\right)=S$. It follows then that $f\left(B_{k_{0}}(f)\right)=S$ for some $0 \leqq k_{0} \leqq q-1$. By the definition of $B_{k_{0}}(f)$, we get that $\operatorname{dim} B_{k_{0}}(f) \geqq m+k_{0}$. This in turn implies that $B_{k_{0}+m}(X) \neq \varnothing$ by virtue of the equality $S_{k_{0}}(f)=S_{k_{0}+m}(X)$ mentioned above. Hence $A \neq \varnothing$. This shows that 3$) \rightarrow 2$ ) in our special case. Conversely, if $A \neq \varnothing$, then $B_{k_{1}}(X) \neq \varnothing$ for some $m \leqq k_{1} \leqq n-1$. Since $B_{k}(X) \subseteq B_{k-m}(f)$ fore very $m$ $\leqq k \leqq n-1$ as follows easily from the definitions of these spaces and the above equality, we have that $\varnothing \neq B_{k_{1}-m}(f) \subseteq A_{f}$. In short, if $S=U$ in 2), then $X$ is reduced.

Next we consider the general case. Let $V$ be any nonempty Zariski open subset of $S$ such that $V$ is nonsingular and $X_{s}$ is generically reduced for every $s \in S$. For example take $V$ to be the set of smooth points of $U$ in case 2) and use Lemma 1.2 in case 3). Then applying the former half of the above consideration to the morphism $\left.f\right|_{X_{V}}: X_{V} \rightarrow V, X_{V}=f^{-1}(V)$, we obtain easily the implication that 3 ) $\rightarrow 2$ ). Conversely assume that 2) holds. Then by the latter half of the above consideration we conclude that $X_{V}$ is reduced. It remains to show that under this condition $X$ is actually reduced. Let $T=f(A) \subseteq S-V$. Suppose that $T \neq \varnothing$ and take a point $t \in T$. Let $H=\{z|| z \mid<1\}$ be the unit disc and $h: H \rightarrow S$ be a morphism of $H$ into $S$ such that $h^{-1}(T)=\{z=0\}$. Let $f_{H}: X_{H} \rightarrow H$ be the induced morphism. Then it is easy to see that $X_{H}$ is not reduced and $X_{H}-h^{-1}(0)$ is reduced contradicting the flatness of $f_{H}$. Hence $T=\varnothing$ and $X$ is reduced.

Q.E D。

Lemma 1. 5. Let $f: X \rightarrow S$ be as in Lemma 1.2. Define $V=\{s$ $\in X ; f$ is flat at every point of $X_{s}$ and $X_{s}$ is reduced $\}$. Then $V$ is a dense Zariski open subset of $S$.

Proof. Let $V_{1}$ be a dense Zariski open subset of $S$ such that $f$ is flat at every point of $X_{s}$. Then by a result of Banica quoted above $V$ is Zariski open in $V_{1}$. Moreover by Lemma 1.2 and the proof of the previous lemma we see easily that it is dense in $S$. Let $T=V_{1}-V$. Then it is enough to show that the closure $\bar{T}$ of $T$ in $S$ is an analytic 
subset of $S$. Let $f: X_{\tilde{S}} \rightarrow \widetilde{S}$ be a flattening of $f$ with the flattening morphism $\phi: \widetilde{S} \rightarrow S$. Then by the above lemma there exists an analytic subset $\widetilde{T}$ of $\widetilde{S}$ such that $X_{\tilde{S}}$ is reduced for $\tilde{s} \in \widetilde{S}$ if and only if $\tilde{s} \in \widetilde{S}-\widetilde{T}$. Then since $\phi$ gives an isomorphism of $\phi^{-1}\left(V_{1}\right)$ and $V_{1}$, it follows that $\phi(\widetilde{T}) \cap V_{1}$ $=T$. Hence $\bar{T}$ is a union of some of the irreducible components of $\phi(\widetilde{T})$ and hence analytic.

Q.E.D.

\section{§. Barlet Space and Its Boundedmess}

2.1. First in 2.1-2.3 we recall some of the constructions due to Barlet [3] of the universal family of compact $q$-cycles on a complex space. Let $S_{k}$ be the symmetric group of degree $k$. For every integer $r \geqq 1$ let $S_{k}$ act on $\left(\mathbb{C}^{r}\right)^{k}$ by the formula $\sigma\left(x_{1}, \cdots, x_{k}\right)=\left(x_{\sigma(1)}, \cdots, x_{\sigma(k)}\right)$, where $x_{i}$ $\in \mathbb{C}^{r}$ and $\sigma \in S_{k}$. Then as is well-known the quotient, $\operatorname{sym}^{k}\left(\mathbb{C}^{r}\right)=\left(\mathbb{C}^{r}\right)^{k} / S_{k}$, has the natural structure of a normal affine algebraic variety. In fact using the elementary symmetric functions of $x_{i}$ Barlet gives a natural embedding, $s: \operatorname{sym}^{k}\left(\mathbb{C}^{r}\right) \rightarrow E$, where $E=\bigoplus_{j=1}^{k} E_{j}$ and $E_{j}$ are the vector spaces of polynomials of degree $j$ on $\mathbb{C}^{r}$.

For $z=\left(z_{1}, \cdots, z_{r}\right) \in \mathbb{C}^{r}$ we put $\|z\|=\sup _{i}\left|z_{i}\right|$. Then define the norm $\|\cdot\|$ on $E$ by $\|P\|=\sup _{\|z\| \leqq 1}|P(z)|, P \in E$. Let $B=B_{R}$ be the polydisc of radius $R>0$ with center at the origin of $\mathbb{C}^{r}$ i.e. $B=\left\{z \in \mathbb{C}^{r} ;\|z\|<R\right\}$. Then $\operatorname{sym}^{k} B=(B)^{k} / S_{k}$ is naturally regarded as an open subset of $\operatorname{sym}^{k}\left(\mathbb{C}^{r}\right)$. In fact there is a suitable relatively compact open subset $U_{R}$ of $E$ such that $\operatorname{sym}^{k} B=s^{-1}\left(U_{R}\right) \quad[3$, p. 16].

Given a polydisc $U$ in $\mathbb{C}^{q}$ and a complex space $Y$ we denote by $H(\bar{U}, Y)$ the space of all the continuous maps $f: \bar{U} \rightarrow Y$ which are holomorphic on $U$, where $\bar{U}$ is the closure of $U$. Then in the above notations, we have the natural inclusions

$$
H\left(\bar{U}, \operatorname{sym}^{k} B\right) \subseteq H\left(\bar{U}, U_{R}\right) \subseteq H(\bar{U}, E) .
$$

Here we note that $H(\bar{U}, E)$ has the natural structure of a Banach space with respect to the sup norm and $H\left(\bar{U}, U_{R}\right)$ is its open subset. Moreover $H\left(\bar{U}, \operatorname{sym}^{k} B\right)$ is a Banach analytic subset of $H\left(\bar{U}, U_{R}\right)$ and in particular has the natural induced topolngy (cf. [3, p. 27 Prop. 4]).

We define the support of an element $f \in H\left(\bar{U}, \operatorname{sym}^{k} B\right)$, denoted by 
$\operatorname{supp} f$, as follows; $\operatorname{supp} f=\left\{(u, b) \in U \times B ; b=b_{1}\right.$ for some $\left(b_{1}, \cdots, b_{k}\right)$ $\left.\in \pi^{-1}(f(u))\right\}$. Here $\pi: B^{k} \rightarrow \operatorname{sym}^{k} B$ is the natural projection. Now suppose that we are given an analytic subset, $X$, of $\mathbb{C}^{q} \times \mathbb{C}^{r}$ defined in a neighborhood of $\bar{U} \times B$. Then we denote by $G_{k, \mathbb{X}}^{U, B}$ the subset of $H\left(\bar{U}, \operatorname{sym}^{k} B\right)$ consisting of those elements whose supports are contained in $X$. This is again a Banach analytic subset of $H\left(\bar{U}, U_{R}\right)[3$, p. 27, Prop. 4].

2. 2. Let $A=\sum_{\alpha=1}^{m} n_{\alpha} A_{\alpha}$ be an effective $q$-cycle defined in a neighborhood of $\bar{U} \times B$. Let $U_{1}$ be any neighborhood of $\bar{U}$ in $\mathbb{C}^{q}$ such that $A$ is defined on $U_{1} \times B$. Then we say that $A$ defines a ramified covering over $U_{1}$, if each $A_{\alpha}$ is finite and surjective over $U_{1}$ under the projection $p_{1}: U_{1} \times B$ $\rightarrow U_{1}$. In this case let $k_{\alpha}$ be the degree of $A_{\alpha}$ over $U_{1}$ and put $k=\sum_{\alpha=1}^{m} k_{\alpha}$. Then we call $k$ the degree of $A$ over $U_{1}$. Now if $A$ is such, then it determines an element

$$
f_{A}^{U} \in H\left(\bar{U}, \operatorname{sym}^{k} B\right)
$$

as follows; let $p_{\alpha}=\left.p_{1}\right|_{A_{a}}: A_{\alpha} \rightarrow U_{1}$ and $U_{1}^{\prime}$ be the dense open subset of $U_{1}$ such that $\left.p_{\alpha}\right|_{p_{\alpha}^{-1}\left(U_{1}^{\prime}\right)}: p_{\alpha}^{-1}\left(U_{1}^{\prime}\right) \rightarrow U_{1}^{\prime}$ is an unramified covering of degree $k_{\alpha}$ for every $\alpha$. Then for every $u \in U_{1}^{\prime}$ we have $p_{\alpha}^{-1}(u)=\left\{\left(u, b_{1}^{\alpha}\right), \cdots\right.$, $\left.\left(u, b_{k_{\alpha}}^{\alpha}\right)\right\}$ for some $b_{j}^{\alpha} \in B$. Then we set $\tilde{f}_{A}^{U}(u)=\pi(b)$ for $u \in U_{1}^{\prime}$, where $b=\left(b_{1}^{1}, \cdots, b_{k_{1}}^{1}, \cdots, b_{1}^{1}, \cdots, b_{k_{1}}^{1}, \cdots, b_{1}^{m}, \cdots, b_{k_{m}}^{m}, \cdots, b_{1}^{m}, \cdots, b_{k_{m}}^{m}\right) \in B^{k}, k_{i}$-tuple $\left(b_{1}^{\alpha}, \cdots, b_{k_{\alpha}}^{\alpha}\right)$ being repeated $n_{\alpha}$ times. Then it is easy to see that $\tilde{f}_{A}^{U}$ extends to a holomorphic map $\tilde{f}_{A}^{U}: U_{1} \rightarrow \operatorname{sym}^{k} B$. Finally we define $f_{A}^{U}$ to be the restriction of $\tilde{f}_{A}^{U}$ to $\bar{U}$. Note that if $|A|$ is further contained in an analytic subspace $X$ in $U_{1} \times B$, then $\operatorname{supp} f_{A}^{U} \subseteq X$ and hence $f_{A}^{U} \in G_{k, X}^{U, B}$.

Conversely, if we are given an element $f \in H\left(\bar{U}, \operatorname{sym}^{k} B\right.$ ) (resp. $\left.G_{k, X}^{U, B}\right)$, then for every polydisc $U_{0}$ with $\bar{U}_{0} \subseteq U$ we can define a unique effective $q$-cycle $A$ (resp. with support in $X$ ) on $U_{0} \times B$ which defines a ramified covering over $U_{0}$ of degree $k$ with $f=f_{A}^{U_{0}}$ (cf. [3, p. 25 Prop. 3]).

Let $X$ be a complex space and $A$ a compact $q$-cycle on $X$. Let $V$ be an open subset of $X$ with an embedding $j: V \rightarrow U_{1} \times B$, where $U_{1}$ (resp. B) is a polydisc in $\mathbb{C}^{q}$ (resp. $\mathbb{C}^{r}$ ). We call such a quadruplet $\left(V, j, U_{1}, B\right.$ ) admissible for $A$ if $j(A \cap V)$ defines a ramified covering over $U_{1}$ in the sense defined above. Thus by the above if $\left(V, j, U_{1}, B\right)$ is admissible for $A$, then to every polydisc $U$ with $\bar{U} \subseteq U_{1}$ we can associate an element 
$f_{A}^{U} \in H\left(\bar{U}, \operatorname{sym}^{k} B\right)$ by $f_{A}^{U}=f_{j(A \cap V)}^{U}$.

Now let $S$ be a reduced complex space and $A_{S}=\left\{A_{s}=\sum n_{\alpha}^{s} A_{a}^{s} ; s \in S\right\}$ a family of compact $q$-cycles on $X$ parametrized by $S$. We call $A_{S}$ analytic if for every admissible quadruplet $\left(V, j, U_{1}, B\right)$ as above and for every polydisc $U$ of $U_{1}$ with $U \subset U_{1}$ the map, $F: S \times U \rightarrow \operatorname{sym}^{k} B$, defined by $F(s, u)=f_{A_{S}}^{U}(u)$ is an analytic map.

Let ( $A n_{\text {red }}$ ) be the category of reduced complex spaces and morphisms. Define a contravariant function $F_{q}:\left(A n_{\text {red }}\right) \rightarrow($ Sets $)$ by $F_{q}(S)=$ the set of analytic families of compact $q$-cycles on $X$ parametrized by $S$. Then the following theorem was shown in [3].

Theorem (Barlet). $F_{q}$ is representable for every $q \geqq 0 . \quad$ Namely there exists a reduced complex space $B_{q}(X)$ and an analytic family, $\left\{A_{b} ; b \in B_{q}(X)\right\}$, of compact $q$-cycles on $X$ parametrized by $B_{q}(X)$, such that every analytic family', $\left\{A_{s} ; s \in S\right\}$, of compact q-cycles on $\mathrm{Y}$ as above is induced by a unique morphism $h: S \rightarrow B_{q}(X)$ from this i.e. $A_{s}=A_{h(s)}$ for all $s \in S$.

We call $B_{q}(X), q \geqq 0$, the Barlet spaces of $X$ and $\left\{A_{b} ; b \in B_{q}(X)\right\}$ the universal families of compact $q$-cycles on $X$. We also write $B(X)$ $=\prod_{q \geq 0} B_{q}(X)$ and call it the Barlet space of $X$.

2. 3. Let $A$ be any compact $q$-cycles on $X$, where $X$ is as in 2.2. Then we may write $A=A_{b_{0}}$ for a unique point $b_{0} \in B_{q}(X)$. We want to describe a system of fundamental neighborhoods of $b_{0}$ in $B_{q}(X)$. First we take a finite open covering $V=\left\{V_{a}\right\}_{1 \leqq \alpha \leqq s}$, of $|A|$ in $X$, with an embedding $j_{\alpha}: V_{\alpha} \rightarrow U_{1 \alpha} \times B_{\alpha}$ of $V_{\alpha}$ into the product of polydiscs $U_{1 \alpha} \subseteq \mathbb{C}^{q_{\alpha}}$ and $B_{\alpha} \subseteq C^{r_{\alpha}}$ for each $\alpha$ such that $\left(V_{\alpha}, j_{a}, U_{1 a}, B_{\alpha}\right)$ is admissible for $A$ in the sense defined in 2.2. Suppose further that there exists for each $\alpha$ an open subset $U_{\alpha}$ of $U_{1 \alpha}$ with $\bar{U}_{\alpha} \subseteq U_{1 \alpha}$, such that $j_{\alpha}^{-1}\left(U_{\alpha} \times B_{\alpha}\right)$ is again an open covering of $|A|$ in $X$. Let us call a set of quintuplets $Q=\left\{\left(V_{\alpha}\right.\right.$, $\left.\left.j_{a}, U_{1 \alpha}, B_{\alpha}, U_{\alpha}\right)\right\}$, with the above property admissible for $A$.

Then to every set of quintuplets admissible for $A$ as above we can associate an element $f_{A}(Q) \in \prod_{\alpha} H\left(\bar{U}_{\alpha}, \operatorname{sym}^{k} B_{\alpha}\right)$ by $f_{A}(Q)=\prod f_{A_{\alpha}}^{U}$. With these preparations we can now state the following lemma which follows 
directly from Barlet's construction [3, Chap. III].

Lemma 2. 且. For every set of quintuplets, $Q=\left\{\left(V_{\alpha}, j_{\alpha}, U_{1 \alpha}, B_{\alpha}\right.\right.$, $\left.\left.U_{\alpha}\right)\right\}_{1 \leqq \alpha \leqq s}$, admissible for $A$ let $W=W(Q)$ be defined by

$$
W=\left\{b \in B_{q}(X) ; Q \text { is admissible for } A_{b}\right\} .
$$

Then $W$ is an open neighborhood of $b_{0}$ in $B_{q}(X)$ and the natural map $\phi=\phi(Q): W \rightarrow \prod_{\alpha} H\left(U_{\alpha}, \operatorname{sym}_{\alpha}^{k} B\right)$, defined by $\phi(b)=f_{A_{b}}(Q)=\prod_{\alpha} f_{A_{b}}^{U_{\alpha}}$, is a homeomorphism onto a finite dimensional analytic subset of $\prod_{\alpha} H\left(U_{\alpha}\right.$, $\left.\operatorname{sym}^{k} B_{\alpha}\right)$. Moreover, $W(Q)$ form a fundamental system of neighborhoods of $b_{0}$ in $B_{q}(X)$ when $Q$ runs through all the set of quintuplets admissible for $A$ as above.

We also need the following local version of the above lemma.

Lemma 2. 2. Let $A=A_{b_{0}}$ be as above. Suppose that $\left(V, j, U_{1}, B\right)$ is a quadruplet admissible for $A$. Then it is also admissible for $A_{b}$ for all $b \in B_{q}(X)$ sufficiently near to $b_{0}$.

2. 4. Recall that every compact $q$-cycle $A$ defines a current $c[A]$ of dimension $2 q$ on $X$ (cf. 1.2). We may now consider $c$ as a map, $c: B_{q}(X)$ $\rightarrow D_{X, 2 n-2 q}^{\prime}$, which is obviously injective and whose image is by definition $C_{q}(X)$.

Proposition 2. 3. The map $c$ gives a homeomorphism of $B_{q}(X)$ onto $C_{q}(X)$, where $C_{q}(X)$ is given the weak topology.

The continuity of $c$ has essentially been proved by Stoll in [23]. Since the situation is not quite the same, however, we shall give here the complete proof following his line. For the proof we need a series of lemmas. In order to avoid interrupting the main line of arguments we defer the proof of the following lemma until the end of this section.

Lemma 2. 4. Let $U_{1}$ (resp. B) be a polydisc in $C^{q}$ (resp. $\mathbb{C}^{r}$ ). Let $A_{0}$ be an effective $q$-cycle and $A_{m}, m \geqq 1$, a family of effective $q$-cycles 
on $U_{1} \times B$. Suppose that for all $m \geqq 0, A_{m}$ are ramified coverings over $U_{1}$ of degree $k$ so that $f_{A_{m}}^{U} \in H\left(\bar{U}, \operatorname{sym}^{k} B\right)$ are defined, where $U$ is any polydisc in $\mathbb{C}^{q}$ with $\bar{U} \subseteq U_{1}$. Suppose further that $f_{A_{m}}^{U}$ converges to $f_{A_{0}}^{U}$ in $H\left(\bar{U}, \operatorname{sym}^{k} B\right)$ as $m \rightarrow \infty$. Let $\left(z_{1}, \cdots, z_{q}\right)$ be the coordinates of $\mathbb{C}^{q}$ and $g$ any continuous function on $\bar{U} \times \bar{B}$. Put $\chi=g d z_{1} \wedge \cdots \wedge d z_{q}$ $\wedge d \bar{z}_{1} \wedge \cdots \wedge d \bar{z}_{q}(\chi=g$, if $q=0)$. Then we have the convergence

$$
\int_{A_{m} \cap(U \times B)} \chi \rightarrow \int_{A_{0} \cap(U \times B)} \chi, m \rightarrow \infty .
$$

To state the next lemma we recall the notion of clear coordinates. Let $W$ be the polydisc of radius $r>0$ in $\mathbb{C}^{n}=\mathbb{C}^{n}\left(z_{1}, \cdots, z_{n}\right)$ and $q \geqq 0$ an integer. Then for every $q$-tuple $I=\left(i_{1}, \cdots, i_{q}\right), 1 \leqq i_{1}<\cdots<i_{q} \leqq n$, let $p_{I}$ : $W \rightarrow W_{I}=\left\{\left(z_{i_{1}}, \cdots, z_{i_{q}}\right) \in \mathbb{C}^{q} ;\left|z_{i_{s}}\right|<r\right\}$ be the natural projection. Let $A$ be an effective $q$-cycle defined in a neighborhood of $\bar{W}$. Then we say that $W$ is clear for $A$ if for every $q$-tuple $I$ as above, the induced map $\left.p_{I}\right|_{|A|}:|A| \rightarrow W_{I}$ is a finite morphism. The following lemma is due to Stoll [23, Prop. 1.3.].

Lemma 2. 5. Let $V$ be a domain in $\mathbb{C}^{n}$ containing the origin, and $A$ an effective $q$-cycle on $V$. Then there exists a unitary (linear) change of coordinates of $\mathbb{C}^{n}$ such that with respect to the new coordinates the polydiscs $W_{r}$ of radius $r$ are clear for $A$ for all sufficiently small $r>0$.

Let $\chi$ be a continuous form of type $(q, q)$ on a domain $V$ of $\mathbb{C}^{n}$ $=\mathbb{C}^{n}\left(z_{1}, \cdots, z_{n}\right)$. We call $\chi$ unmixed with respect to the coordinates $\left(z_{1}\right.$, $\left.\cdots, z_{n}\right)$ if $\chi$ is written on $V$ in the form $\chi=\sum_{I} \chi_{I} d z_{i_{1}} \wedge d \bar{z}_{i_{1}} \wedge \cdots \wedge d z_{i_{q}} \wedge d \bar{z}_{i_{q}}$, where $\chi_{I}$ are continuous functions on $V$ and $I$ runs through all the $q$ tuples $I=\left(i_{1}, \cdots, i_{q}\right)$ with $1 \leqq i_{1}<\cdots<i_{q} \leqq n$. Using Lemma 2.5 the following two lemmas can be proved quite in the same way as Lemma 3.4 and 3.6 respectively in [23].

Lemma 2.6. Let $W=\left\{\left(z_{1}, \cdots, z_{n}\right) \in \mathbb{C}^{n} ;\left|z_{i}\right|<r\right\}$ be the polydisc of radius $r$ in $\mathbb{C}^{n}$. Let $A_{0}$ be an effective q-cycle (resp. $A_{m}, m \geqq 1$, a family of effective q-cycles) defined in a neighborhood of $\bar{W}$ with 
$q>0$. Suppose that $W$ is clear for all the $A_{m}, m \geqq 0$. For every $q$ tuple $I=\left(i_{1}, \cdots, i_{q}\right), 1 \leqq i_{1}<\cdots<i_{q} \leqq n$, we can then define elements $f_{A_{m}}^{W_{I}}$ $\in H\left(\bar{W}_{I}, W_{I^{\prime}}\right)$, where $I^{\prime}$ is the $(n-q)$-tuple complementary to $I$. Suppose that for every $I, f_{A_{m}}^{W_{I}}$ converges to $f_{A_{0}}^{W_{I}}$ as $m \rightarrow \infty$. Then for every continuous unmixed form, $\chi$, of type $(q, q)$ defined on $\bar{W}$ we have the convergence

$$
\int_{A_{m} \cap W} \chi \rightarrow \int_{A_{0} \cap W} \chi, m \rightarrow \infty
$$

Lemma 2.7. Let the notations and assumptions be the same as in Lemma 2.5. Suppose further that $\left|A_{0}\right|=U_{1} \times\{0\}$ and $q>0$. Then for every continuous form $\chi$ defined on $U_{1} \times \bar{B}$ we have the convergence

$$
\int_{(U \times B) \cap A_{m}} \chi \rightarrow \int_{(U \times B) \cap A_{0}} \chi, m \rightarrow \infty .
$$

Next we give two lemmas needed in the proof of the continuity of $c^{-1}$.

Lemma 2. 8. Let $V$ be a domain in $\mathbb{C}^{n}$ containing the origin. Let $A_{0}$ be an effective $q$-cycle on $V$ and $A_{m}, m \geqq 1$, a family of effective q-cycles on $V$. Suppose that $c\left[A_{m}\right]$ converges weakly to $c\left[A_{0}\right]$ as $m \rightarrow \infty$. Then there exists a linear subspace, $P$, of complex dimension $n-q$ of $\mathbb{C}^{n}$ such that if $P^{\prime}$ is the linear subspace orthogonal to $P$ with respect to the standard Hermitian metric on $\mathbb{C}^{n}$, then for some polydiscs $U_{1}$ of $P^{\prime}$ and $B$ of $P, U_{1} \times B$, considered naturally as a subdomain of $\mathbb{C}^{n}$, is admissible for $A_{n}$ for all $n \geqq 0$.

Proof. For $q=0$ and $q=n$ the assertion is trivial. So we assume that $0<q<n$. For any subset $E_{0}$ of $V$ let $h^{k}\left(E_{0}\right)$ be the $k$-dimensional Hausdorf measure of $E_{0}$ (cf. $[15,2.4]$ ). Set $E=\bigcup_{n \geq 0}\left|A_{n}\right|$. Then $h^{2 q+1}$. $(E) \leqq \sum_{n \geqq 0} h^{2 q+1}\left(\left|A_{n}\right|\right)=0$. Hence by $[20$, Lemma 4. 2.] there exists a complex linear subspace, $P$, of dimension $n-q$ of $\mathbb{C}^{n}$ such that $P \cap E$ is totally disconnected. Thus to finish the proof it suffices, up to the usual arguments, to show that $E$ is closed in $V$. So let $x$ be a point in the closure, $\bar{E}$, of $E$ in $V$ with the sequence, $\left\{x_{n}\right\}$, of points of $E$ converging to $x$. Suppose now that $x \notin E$. Take for each $k$ an $m_{k} \geqq 0$ such that 
$x_{k} \in\left|A_{m_{k}}\right|$. We may assume that $m_{k} \rightarrow \infty$ for $k \rightarrow \infty$. For any point $a$ $\in \mathbb{C}^{n}$ let $B_{r}(a)$ be the ball of radius $r$ with center $a$. Then take $r>0$ sufficiently small so that $B_{r}(x) \subseteq V$ and $B_{r}(x) \cap A_{0}=\phi$. Let $\rho$ be a $C^{\infty}$ function with compact support in $V^{r}$ and $\equiv 1$ on $B_{r}(x)$. Put $\chi=\rho v_{q}$ with the volume $2 q$-form $v_{q}$ (cf. 1.2). Then by our assumption $\int_{A_{m_{k}}} \chi \rightarrow \int_{A_{0}} \chi$ $=0$. On the other hand, for sufficiently large $k, B_{r / 2}\left(x_{k}\right) \subseteq B_{r}\left(x_{0}\right)$. Hence $\int_{A_{m_{k}}} \chi \geqq \int_{A_{m_{k} \cap B_{r / 2}\left(x_{k}\right)}} \chi=\int_{A_{m_{k} \cap B_{r / 2}\left(x_{k}\right)}} v_{q}$, while by [4, Lemma 3] $\int_{A_{m_{k} \cap B_{r / 2}\left(x_{n}\right)}} v_{q}$ $\geqq c(r / 2)^{2 q}$ for some positive constant $c$ independent of $k$. This is a contradiction. Hence $E=\bar{E}$ as desired.

Q.E.D.

Lemma 2.9. Let $U_{1}$ and $U$ (resp. $B_{1}$ and $B$ ) be polydiscs in $\mathbb{C}^{\prime}$ (resp. $\mathbb{C}^{r}$ ) with $\bar{U} \subseteq U_{1}$ (resp. $\bar{B} \subseteq B_{1}$ ). Let $A_{m}, m \geqq 1$, be a family of effective q-cycles on $U_{1} \times B$. Suppose that $A_{m}$ are ramified coverings over $U_{1}$ of a fixed degree $k$ so that in particular the associated elements $f_{A_{m}}^{U} \in H\left(\bar{U}, \operatorname{sym}^{k} B\right)$ can be defined. Then there exists an effective q-cycle $A_{0}$ in $\bar{U} \times B$ and a subsequence $\left\{m_{j}\right\}$ of $\{m\}$ such that $A_{0}$ is a ramified covering over $\bar{U}$ of degree $k$ with that associated element $f_{A_{0}}^{U} \in H\left(\bar{U}, \operatorname{sym}^{k} B_{1}\right)$ and that $f_{A_{m_{1}}}^{U}$ converges to $f_{A_{0}}^{U}$ in $H(\bar{U}$, $\left.\operatorname{sym}^{k} B_{1}\right)$ for $j \rightarrow \infty$.

Proof. We regard $\operatorname{sym}^{k} B_{1}$ as an analytic subset of an open set in $E$ as in 2.1. Then with respect to some basis of $E$ each $f_{A_{m}}^{U}$ is represented by an $N$-tuple of bounded holomorphic functions $\left(f_{m 1}, \cdots, f_{m N}\right)$ defined on $\bar{U}$, where $N=\operatorname{dim} E$. Since $A_{m}$ are all defined on $U_{1} \times B_{1}$ we may assume that $f_{m i}$ are defined and holomorphic on a polydisc $U^{\prime} \supset \bar{U}$. Then by the generalized Vitali's theorem [11, I. A 12] we can find a subsequence $\left\{m_{j}\right\}$, of $\{m\}$ such that for each $i f_{m, i}$ converges to a holomorphic function $f_{i}$ defined on $U^{\prime}$ uniformly on $\bar{U}$. Then for the map $F: \bar{U} \rightarrow \mathbb{C}^{N}$ defined by $F=\left(f_{i}\right), F(\bar{U}) \subseteq \overline{\operatorname{sym}^{k} B} \subseteq \operatorname{sym}^{k} B_{1}$. Then as was remarked in 2.2 there exists an effective $q$-cycle $A_{0}$ as in the lemma such that $F$ has the form $F=f_{A_{0}}^{U}$ as an element of $H\left(\bar{U}, \operatorname{sym}^{k} B_{1}\right)$.

Q.E.D.

Proof of Proposition 2.3. 1) continuity of $c$. Let $b_{n}, m=1,2, \cdots$, be any sequence of points of $B_{q}(X)$ converging to a point $b_{0} \in B_{q}(X)$. Let $A_{m}=A_{b_{m}}, m \geqq 0$, be the corresponding compact $q$-cycles on $X$. Let 
$\chi$ be any continuous form with compact support on $X$. Then it is enough to show that for $m \rightarrow \infty$ we have the convergence

$$
c\left[A_{m}\right](\chi)=\int_{A_{m}} \chi \rightarrow c\left[A_{0}\right](\chi)=\int_{A} \chi
$$

Note that since $A_{m}$ are holomorphic cycles, we may here assume that $\chi$ is of type $(q, q)$. Based on the above lemmas the proof of $\left(^{*}\right)$ is formally quite analogous to that of Stoll in [23] as mentioned above (cf. the arguments in Lemma 2.3 Theorem 3.8 in [22]). Now the assertion follows immediately from Lemma 2.4 if $q=0$. So we assume that $q>0$. Take and fix any $\varepsilon>0$. Let $\left|A_{0}\right|_{\text {sing }}$ be the set of singular points of $\left|A_{0}\right|$. Consider first the following assertion. (**) We can find an open covering $\{U, V\}$ of $X$ with the following property; let $\left\{\lambda_{U}, \lambda_{V}\right\}$ be any partition of unity subordinate to the covering $\{U, V\}$. Then we have

a) $\quad \int_{A_{m} \cap U}\left|\lambda_{U} \chi\right|<\varepsilon / 3$ for $m=0$ and $m \gg 0$ and

b) $\left|\int_{A_{m} \cap V} \lambda_{V} \chi-\int_{A_{0} \cap V} \lambda_{V} \chi\right|<\varepsilon / 3$ for $m \gg 0$.

We first see that (**) implies (*). Indeed, we have for $m \gg 0$ $\left|\int_{A_{m}} \chi-\int_{A_{0}} \chi\right| \leqq \int_{A_{m} \cap U}\left|\lambda_{U} \chi\right|+\int_{A_{0} \cap U}\left|\lambda_{U} \chi\right|+\left|\int_{A_{m} \cap V} \lambda_{V} \chi-\int_{A_{0} \cap V} \lambda_{V} \chi\right|<\varepsilon$. This proves $\left(^{*}\right)$. Here $\int_{A_{0} \cap V}\left|\lambda_{U} \chi\right|=\sum_{\beta} n_{\beta} \int_{A_{\beta}}\left|\iota_{\beta}^{*} \lambda_{U} \chi\right|$, where $A_{0}=\sum n_{\beta} A_{\beta}$ and $\iota_{\beta}: A_{\beta} \cap U \rightarrow U$ is the inclusion, and similarly for $\int_{A_{m} \cap U}\left|\lambda_{U} \chi\right|$ (cf. 1.3). We use the similar notations also in the following.

Now we show $\left.{ }^{* *}\right)$. First we show the existence of a neighborhood $U$ of $\left|A_{0}\right|_{\text {sing }}$ with the property a) above. We take and fix finite open coverings $\mathfrak{B}^{\prime}=\left\{V_{\alpha}^{\prime}\right\}$ and $\mathfrak{Z}=\left\{V_{\alpha}\right\}, \alpha=1, \cdots, k$, of $\left|A_{0}\right|_{\text {sing }}$ in $X$ with $\bar{V}_{\alpha}$ $\subset V_{\alpha}^{\prime}$ such that for each $\alpha$ there are 1) an embedding $j_{\alpha}^{\prime}: V_{\alpha}^{\prime} \rightarrow D_{\alpha}^{\prime}$ of $V_{\alpha}^{\prime}$ into a domain $D_{\alpha}^{\prime}$ in $\mathbb{C}^{n_{\alpha}}$,2) relatively compact subdomain, $D_{\alpha}$, of $D_{\alpha}^{\prime}$ with $j_{\alpha}^{-1}\left(D_{\alpha}\right)=V_{\alpha}$ and 3$)$ a continuous $2 q$-form, $\tilde{\chi}_{\alpha}$, of type $(q, q)$ on $D_{\alpha}^{\prime}$ with $j_{\alpha}^{*} \tilde{\chi}_{\alpha}=\left.\chi\right|_{v_{\alpha}}$. Let $\left\{\varphi_{\alpha}\right\}$ be any partition of unity subordinate to the covering $\mathfrak{W}$ and for each $\alpha$ take and fix a continuous function $\widetilde{\varphi}_{\alpha}$ on $D_{\alpha}^{\prime}$ with support in $D_{\alpha}, 0 \leqq \widetilde{\varphi}_{\alpha} \leqq 1$, and with $j_{\alpha}^{*} \widetilde{\varphi}_{\alpha}=\varphi_{a}$. Let $\left(z_{1}^{\alpha}, \cdots, z_{n}^{\alpha}\right)$ be the coordinates of $\mathbb{C}^{n_{\alpha}}$ and form the volume $2 q$ form $v_{q, \alpha}=$ $(\sqrt{-1} / 2)^{q} \sum_{I} d z_{i_{1}}^{\alpha} \wedge d \bar{z}_{i_{1}}^{\alpha} \wedge \cdots \wedge d z_{i_{q}}^{\alpha} \wedge d \bar{z}_{i_{q}}^{\alpha}$ as in 1.2. Then there exists a constant $c_{\alpha}>0$ such that $\left|\widetilde{\varphi}_{\alpha} \tilde{\chi}_{\alpha}\right| \leqq c_{\alpha} v_{q, \alpha}$ on $D_{\alpha}$ (cf. 1.3.3)). Next take and fix 
a continuous function $g_{a}$ with compact support on $D_{\alpha}^{\prime}$ such that $0 \leqq g_{\alpha} \leqq 1$ and $g_{\alpha} \equiv 1$ on $\bar{D}_{\alpha}$. Then obviously we have $\left|\widetilde{\varphi}_{\alpha} \tilde{\chi}_{\alpha}\right| \leqq c_{\alpha} g_{\alpha} v_{q, \alpha}$.

Now writing $A_{m, \alpha}=A_{m} \cap V_{\alpha}$ and $\tilde{A}_{m, \alpha}=j_{\alpha}\left(A_{m, \alpha}\right)$ we consider the following assertion: (") For each $\alpha$ there exists a neighborhood $\widetilde{U}_{\alpha}$ of $\left|\widetilde{A}_{0, \alpha}\right|_{\text {sing }}$ in $D_{\alpha}^{\prime}$ such that $\int_{\tilde{A}_{0, \alpha} \cap \widetilde{U}_{\alpha}} g_{\alpha} v_{q, \alpha}<\varepsilon / 6 k c_{\alpha}$ and $\mid \int_{\tilde{A}_{0, \alpha} \cap \widetilde{U}_{a}} g_{a} v_{q, \alpha}$ $-\int_{\tilde{A}_{m, \alpha} \cap \widetilde{U}_{\alpha}} g_{\alpha} v_{q, \alpha} \mid<\varepsilon / 6 k c_{\alpha}$ for $m \gg 0$. Note that from these inequalities it follows immediately that $\int_{\tilde{A}_{m, \alpha} \cap \tilde{U}_{\alpha}} g_{\alpha} v_{q, \alpha}<\varepsilon / 3 k c_{\alpha}$ for $m=0$ and $m \gg 0$. We shall see that (") implies the existence of $U$. In fact let $U_{\alpha}=j_{\alpha}^{-1}\left(\widetilde{U}_{\alpha}\right)$

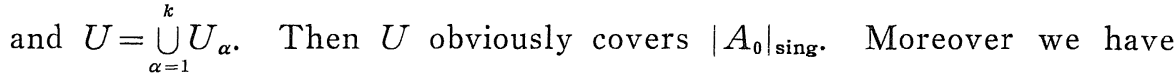
that

(1) $\int_{A_{m, \alpha} \cap U_{\alpha}}\left|\varphi_{\alpha} \chi_{\alpha}\right|=\int_{\tilde{A}_{m, \alpha} \cap \widetilde{U}_{\alpha}}\left|\widetilde{\varphi}_{\alpha} \tilde{\chi}_{\alpha}\right| \leqq c_{\alpha} \int_{\tilde{A}_{m^{\prime} \alpha} \cap \widetilde{U}_{\alpha}} g_{\alpha} v_{q, \alpha}<\varepsilon / 3 k$ and hence $\int_{A_{m} \cap U}\left|\lambda_{U} \chi\right| \leqq \int_{A_{m} \cap U}|\chi|=\int_{A_{m} \cap U}\left|\sum \varphi_{\alpha} \chi_{\alpha}\right| \leqq \sum_{\alpha=1}^{k} \int_{A_{m}, \alpha \cap U_{\alpha}}^{k}\left|\varphi_{\alpha} \chi_{\alpha}\right|<\varepsilon / 3$ for $m=0$ and $m \gg 0$, which is a) of $(* *)$.

We turn to prove ("). We take and fix one $\alpha$ for the moment and suppress the suffix $\alpha$. Set $C=\operatorname{supp}\left(g v_{q}\right) \cap\left|\tilde{A}_{0}\right|_{\text {sing, }}$, where supp denotes the support. Then $C$ is compact in $D^{\prime}$. Hence we can take a neighborhood $\left|\widetilde{U}_{0}\right|$ of $\left|\widetilde{A}_{0}\right|_{\text {sing }}$ in $D^{\prime}$ such that $\int_{\tilde{A}_{0} \cap U} g v_{q}<\varepsilon / 6 k c_{\alpha}$. Next we take a finite open covering $\left\{\widetilde{U}_{1}, \cdots, \widetilde{U}_{r}\right\}$ of $C$ in $D^{\prime}$ such that each $\widetilde{U}_{i}$ is clear for $\tilde{A}_{0}$ possibly after a unitary linear change of coordinates. The existence of such $\widetilde{U}_{i}$ follows immediately from Lemma 2.5. Then by Lemma $2.2 \widetilde{U}_{i}$ are also clear for $A_{m}$ for $m \gg 0$. Note that we may assume that $\widetilde{U}_{i}$ are all contained in $\widetilde{U}_{0}$. Let $\left\{\rho_{i}\right\}$ be the partition of unity subject to the covering $\left\{\widetilde{U}_{i}\right\}$. Finally let $\widetilde{U}^{\prime}$ be a neighborhood of $\left|\tilde{A}_{0}\right|_{\text {sing }}$ such that $\widetilde{U}^{\prime} \cap \operatorname{supp} g v_{q} \subseteq \bigcup_{i=1}^{r} \widetilde{U}_{i}$, and put $\widetilde{U}=\widetilde{U}^{\prime} \cup\left(\bigcup_{i=1}^{r} \widetilde{U}_{i}\right)$. Then this $\widetilde{U}$ satisfies the condition of $\left(^{\prime \prime}\right)$. The proof is quite analogous to (1) once we note the following; by Lemma 2.5 and the fact that any unitary change of coordinates leaves invariant $v_{q}$ we have for any $\eta>0$ $\left|\int_{\tilde{A}_{m} \cap \tilde{U}} \rho_{i} g v_{q}-\int_{\tilde{A}_{0} \cap U_{i}} \rho_{i} g v_{q}\right|<\eta$ for $m \gg 0, \quad i=1, \cdots, r$, and obviously $\int_{\widetilde{U} \cdot-\cup \widetilde{v}_{i}} g v_{q}=0$. Hence we omit it.

It remains to find $V \subseteq X$ such that together with $U$ it satisfies the conditions of $\left(^{* *}\right)$. Let $U^{\prime}$ be a neighborhood of $\left|A_{0}\right|_{\text {sing }}$ with $\bar{U}^{\prime} \subseteq U$ and $V^{\prime}=X-U^{\prime}$. Let us take a set of quintuplets $\left(V_{\alpha}, J_{\alpha}, U_{1 \alpha}, B_{\alpha}, U_{\alpha}\right)$, 
$\alpha=1, \cdots, d^{\prime}$, admissible for $\left|A_{0}\right|$ with the following properties; for $\alpha=1$, $\cdots, d, 1) \quad V_{\alpha}^{0}=j_{\alpha}^{-1}\left(U_{\alpha} \times B_{\alpha}\right)$ covers $V^{\prime}$ and $V_{\alpha} \cap\left|A_{0}\right|_{\text {sing }}=\varnothing$ and 2) $j_{\alpha}\left(V_{\alpha}\right.$ $\left.\cap\left|A_{0}\right|\right)=U_{1} \times\{0\}$. Put $V=\bigcup_{\alpha=1}^{d} V_{\alpha}^{0}$. Then clearly $\left|A_{0}\right| \subseteq U \cup V$. Let $\left\{\lambda_{U}\right.$, $\left.\lambda_{v}\right\}$ be as in $\left(^{* *}\right)$ and $\left\{\psi_{\alpha}\right\}$ be the partition of unity subordinate to $\left\{V_{\alpha}^{0}\right\}$. We may assume that for every $\alpha$ we are given a continuous $2 q$-form of type $(q, q), \tilde{\chi}_{\alpha}$, (resp. continuous functions $\tilde{\lambda}_{U, \alpha}, \tilde{\lambda}_{V, \alpha}, \tilde{\psi}_{\alpha}$ ) such that $j_{\alpha}^{*} \tilde{\chi}_{\alpha}=\left.\chi\right|_{V_{\alpha}}\left(\operatorname{resp} . j_{\alpha}^{*} \tilde{\lambda}_{U, \alpha}=\left.\lambda_{U}\right|_{V_{\alpha}}, j_{\alpha}^{*} \tilde{\lambda}_{V, \alpha}=\left.\lambda_{V}\right|_{V_{\alpha}}, j^{*} \tilde{\psi}_{\alpha}=\psi_{\alpha}\right.$ ). Then by Lemma 2.7 we have $\left|\int_{\tilde{A}_{m, \alpha}} \tilde{\psi}_{\alpha} \tilde{\lambda}_{V, \alpha} \tilde{\chi}_{\alpha}-\int_{\tilde{A}_{0, \alpha}} \tilde{\psi}_{\alpha} \tilde{\lambda}_{V, \alpha} \tilde{\chi}_{\alpha}\right|<\varepsilon / 3 d$ for $m \gg 0$. From this b) follows easily as (1). This completes the proof of (**) and hence the continuity of $c$.

2) The continuity of $c^{-1}: C_{q}(X) \rightarrow B_{q}(X)$. Suppose that $c\left[A_{b_{n}}\right], n \geqq 1$, converges weakly to $c\left[A_{b_{0}}\right], b_{n} \in B_{q}(X), n \geqq 0$. We show that $b_{n}$ converges to $b_{0}$ in $B_{q}(X)$. Write $A_{n}=A_{b_{n}}, n \geqq 0$. First for every point $x \in X$ we shall find a quadruplet $\left(V, j, U_{1}, B\right)$ admissible for $A_{n}$ for all $n \geqq 0$ and with $x \in V$ (cf. 2.2). Fix a neighborhood $W$ of $x$ and an embedding $j: W \rightarrow G$, where $G$ is a domain in $\mathbb{C}^{m}$. We write $A_{n}(j)=j\left(W \cap A_{n}\right)$. Then by Lemma 2.8 after a suitable linear change of coordinates of $\mathbb{C}^{m}$ we can find polydiscs $U_{1} \subseteq \mathbb{C}^{q}$ and $B \subseteq \mathbb{C}^{r}$ such that $U_{1} \times B$ is relatively compact in $G$ and $A_{n}(j)$ are all ramified coverings over $U_{1}$, where $m=q+r$ and $\mathbb{C}^{m}=\mathbb{C}^{q} \times \mathbb{C}^{r}$. Let $k_{n}$ be the degree of $A_{n}$ over $U_{1}$. Let $\left(z_{1}, \cdots, z_{q}\right)$ be the coordinates of $\mathbb{C}^{q}$ and put $\chi=(\sqrt{-1})^{q} \rho d z_{1} \wedge \cdots \wedge d z_{q} \wedge d \bar{z}_{1} \wedge \cdots \wedge d \bar{z}_{q}$ considered as a $(q, q)$-form on $U_{1} \times B$ and on $U_{1}$ alternatively according to the occasions, where $\rho$ is a non-negative continuous function on $U_{1}$ with support in $U_{1}$ and $\not \equiv 0$. Then we have $\int_{A_{n}(j)} \chi=k_{n} \int_{V_{1}} \chi \neq 0$. On the other hand, since $\int_{A_{n}(j)} \chi \rightarrow \int_{A_{0}(j)} \chi$ for $n \rightarrow \infty, k_{n}=k$ for $n \gg 0$. If we put $V=j^{-1}\left(U_{1} \times B\right)$, then $\left(V, j, U_{1}, B\right)$ has the desired property。

Now for every point $x \in X$ take such a quadruplet $\left(V_{x}, j_{x}, U_{1 x}, B_{x}\right)$ and a polydisc $U_{x}$ in $U_{1 x}$ with $\bar{U}_{x} \subseteq U_{1 x}$. Take a finite number of points $x_{1}, \cdots, x_{d} \in X$ such that $j_{x_{i}}^{-1}\left(\bar{U}_{x_{i}} \times B_{x_{i}}\right)$ covers $\left|A_{0}\right|$. Then $\left(V_{x_{i}}, j_{x_{i}}, U_{1 x_{i}}\right.$, $\left.B_{x_{i}}, U_{x_{i}}\right)$ is an admissible set of quintuplets for $A_{0}$ and $A_{n}$ for $n \gg 0$ (cf. 2.3). Then by Lemma 2.1 it is enough to show that for each $i, f_{A_{n}}^{U_{x_{i}}}$ converges to $f_{A_{0}}^{U_{x_{i}}}$ in $H\left(\bar{U}_{x_{i}}, \operatorname{sym}^{k_{i}} B_{x_{l}}\right)$, where $k_{i}$ is the degree of $A_{0}$ over $U_{1 x_{i}}$. To show this we drop the suffix $x_{i}$. Suppose the contrary so that there exists a neighborhood $N$ of $f_{A_{0}}^{U}$ in $H\left(\bar{U}, \operatorname{sym}^{k} B\right)$ and a 
subsequence $\left\{n_{k}\right\}$ of $\{n\}$ such that $f_{A_{n_{k}}}^{U} \notin N$ for all $k$. By Lemma 2.9 there exists a convergent subsequence of $\left\{n_{k}\right\}$, which we may assume to be $\left\{n_{k}\right\}$ itself, such that $f_{A_{n_{k}}}^{U}$ converges to $f_{A_{n_{0}}}^{U}$ in $H\left(\bar{U}, \operatorname{sym}^{k} B^{\prime}\right)$ for a larger polydisc $B^{\prime} \supseteqq B$. Taking $B^{\prime}$ instead of $B$ we may assume that $B=B^{\prime}$. Then by the continuity of $c$ established above $A_{n_{k}}(j)$ converges weakly to $A_{n_{0}}(j)$ as well as to $A_{0}(j)$. Thus $A_{n_{0}}(j)=A_{0}(j)$, since the limit is unique. This is a contradiction and hence the proof is complete.

We call a subset $E \subseteq B_{q}(X)$ bounded if $c(E)$ is bounded in $C_{q}(X)$ in the sense of Definition 1.1.

Proposition 2. 10.3) Let $E$ be a bounded subset of $B_{q}(X)$ such that $\underset{\in E}{\cup}\left|A_{b}\right|$ is contained in a compact subset $K$ of $X$. Then $E$ is relatively compact in $B_{q}(X)$.

For the proof we need the following deep result of Harvey and Shiffman [12, Theorem 3.9].

Theorem (Harvery and Shiffman). Let $V$ be a domain in $\mathbb{C}^{n}$. Let $\left\{A_{n}\right\}, n \geqq 1$, be a bounded family of effective $q$-cycles on $V$. Then there is a subsequence of $\left\{A_{n}\right\}$ which converges either to an effective q-cycle $A_{0}$ on $V$ or to 0 with respect to the weak topology. Further if there is a point $x \in V$ which is a limit point of the sequence $\left\{x_{n}\right\}$ with $x_{n} \in\left|A_{n}\right|$, then the above limit is not 0 and in fact $x \in\left|A_{0}\right|$.

For the last statement see the proof of [12, Theorem 3.10] or of Lemma 2.8 above.

Proof of Proposition 2.10. It is easy to globalize the above theorem of Harvey and Shiffman to obtain the fact that every bounded sequence $\left\{c\left[A_{n}\right]\right\}$ in $C_{q}(X)$ has a subsequence which converges either to an effective $q$-cycle $A_{0}$ on $X$ or to 0 . Now suppose that $A_{n} \in E$ for all $n$. Take a point $x_{n} \in A_{n}$ for each $n$. Then there is a subsequence of $\left\{x_{n}\right\}$ converging to a point $x \in K$ since $K$ is compact. Then by the above theorem

3) cf. 2). 
the limit is a $q$-cycle $A_{0}$. Finally since $\left|A_{0}\right| \subseteq K, A_{0}$ is compact. This shows the relative compactness of the set $C(E)$, since $C_{q}(X)$ has countable topology. Then the proposition follows from Proposition 2.3.

Q.E.D.

We end this section with the proof of Lemma 2.4. Let $U_{1}, B, U, g$ and $\chi$ be as in the lemma. First in general let $A=\sum_{\alpha=1}^{m} n_{\alpha} A_{\alpha}$ be an effective $q$-cycle on $U_{1} \times B$ which is a ramified covering of degree $k$ over $U_{1}$ as in 2. 2. Let $f_{A}^{U} \in H\left(\bar{U}, \operatorname{sym}^{k} B\right)$ be the corresponding element. Then we define a continuous function on $\bar{U}, \operatorname{Tr}_{A} g$, called the trace of $g$ with respect to $A$, as follows. First define a continuous function $\widetilde{g}$ on $\bar{U} \times(B)^{k}$ by $\widetilde{g}\left(u,\left(b_{1}, \cdots, b_{k}\right)\right)=\sum_{i=1}^{m} g\left(u, b_{i}\right)$. Then since $g$ is invariant under the natural action of the symmetric group $S_{k}$ on $\bar{U} \times(B)^{k}$, there is a unique continuous function $\bar{g}$ on $\bar{U} \times \operatorname{sym}^{k} B$ such that $\widetilde{g}=\bar{\pi}^{*} \bar{g}$, where $\bar{\pi}=i d_{\bar{U}} \times \pi$ : $\bar{U} \times B^{k} \rightarrow \bar{U} \times \operatorname{sym}^{k} B$ with $\pi:(B)^{k} \rightarrow \operatorname{sym}^{k} B$ the natural projection. Finally we put

$$
\left(\operatorname{Tr}_{A} g\right)(u)=\bar{g}\left(u, f_{A}^{U}(u)\right)
$$

Note that $\operatorname{Tr}_{A} g$ depends only on the restriction of $g$ on $|A|$.

Set $\operatorname{Tr}_{A} \chi=\operatorname{Tr}_{A} g d z_{1} \wedge \cdots \wedge d z_{q} \wedge d \bar{z}_{1} \wedge \cdots \wedge d \bar{z}_{q}$. Then we show that

$$
\int_{A} \chi=\int_{U} \operatorname{Tr}_{A} \chi
$$

For this we go back to the definition of $f_{A}^{U}$ in 2.2. Let $U_{1}^{\prime}$ be as in 2. 2. Then for every $u \in U_{1}^{\prime} \cap \bar{U}$ we have $f_{A}^{U}(u)=\pi(b)$ with $b=\left(b_{1}^{1}, \cdots\right.$, $\left.b_{k_{1}}^{1}, \cdots, b_{1}^{m}, \cdots, b_{k_{m}}^{m}\right)$ in the notation there. Hence if $u \in U_{1}^{\prime} \cap \bar{U}$, it follows that $\left(\operatorname{Tr}_{A} g\right)(u)=\bar{g}\left(u, f_{A}^{U}(u)\right)=g(u, \pi(b))=\sum_{a=1}^{m} n_{\alpha} \sum_{j=1}^{k_{\alpha}} g\left(u, b_{j}^{a}\right)$. Hence $\int_{U} \operatorname{Tr}_{A} \chi=\int_{U^{\prime} \cap \cup} \operatorname{Tr}_{A} \chi=\sum_{\alpha} n_{\alpha} \int_{U^{\prime} \cap \cup U} \sum_{j} g\left(u, b_{j}^{\alpha}\right) d z_{1} \wedge \cdots \wedge d z_{q} \wedge d \bar{z}_{1} \wedge \cdots \wedge d \bar{z}_{q}$. on the other hand, since $\left.p_{\alpha}\right|_{p_{\alpha}^{-1}\left(U_{1}^{\prime}\right)}: p_{\alpha}^{-1}\left(U_{1}^{\prime}\right) \rightarrow U_{1}^{\prime}$ is unramified, it easily follows that

$$
\int_{U_{1}^{\prime} \cap U} g\left(u, b_{j}^{\alpha}\right) d z_{1} \wedge \cdots \wedge d \bar{z}_{q}=\int_{A_{\alpha} \cap\left(U_{1}^{\prime} \cap U\right) \times B} \chi=\int_{A_{\alpha}} \chi
$$

Then combining the above two equalities we get (2).

Now we come back to the proof of Lemma 2.4. By assumption $f_{A_{m}}^{U}$ 
converges uniformly on $\bar{U}$ to $f_{A_{0}}^{U}$. Then by the definition of $\operatorname{Tr}_{A} g, \operatorname{Tr}_{A_{m}} g$ converges uniformly on $\bar{U}$ to $\operatorname{Tr}_{A_{0}} g$. Then the lemma follows immediately from (2) by the definition of $\operatorname{Tr}_{A} \gamma$.

\section{$\S$ 3. Property $\mathbb{B} \mathbb{P}$ and $\overline{\mathbb{D}} \mathbb{P}$}

3.1. We recall the definition of the multiplicity of a complex space. Let $\mathrm{Y}$ be an irreducible complex space, i.e. $Y_{\text {red }}$ is irreducible, and $q$ $=\operatorname{dim} Y$. Then we can find a Zariski open subset $U$ of $Y$ and an integer $m \geqq 1$ with the following property: For every point $x \in U$ let $j=j_{x}: U_{x}$ $\rightarrow V_{x}$ be any embedding of a neighborhood $U_{x}$ of $x$ in $U$ into a domain $V_{x}$ in $\mathbb{C}^{q+r_{x}}$ such that $j\left(U_{x}\right)_{\text {red }}=V_{x} \cap\left(\mathbb{C}^{q} \times\{0\}\right)$. Then if $\pi: j\left(U_{x}\right) \rightarrow j\left(U_{x}\right)_{\text {red }}$ is the map induced by the natural projection $\pi_{0}: \mathbb{C}^{q+r_{x}} \rightarrow \mathbb{C}^{q}$, then $\mathcal{O}_{j(U), x}$ is a

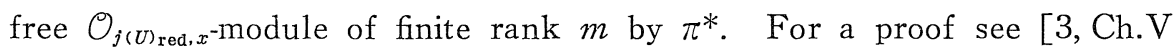
$\S 1]$. The integer $m$ is clearly independent of $U$ and hence an invariant of $Y$. We call $m=m_{Y}$ the multiplicity of $Y$.

Let $X$ be a complex space and $Y$ a compact subspace of $X$ of pure dimension $q$. Let $\mathcal{G}$ be the ideal sheaf of $Y$ in $X$ and $\mathcal{I}=\cap Q_{i}$ be any irredundant primary decomposition of $\mathcal{G}$ (cf. [22]). Let $Y_{i}$ be the subvariety of $X$ corresponding to $Q_{i}$, and $m_{i}$ the multiplicity of the irreducible complex space $\left(Y_{i}, \mathcal{O}_{x} / Q_{i}\right)$ defined above. Then we define a compact $q$-cycle $[Y]$ by,

$$
[Y]=\sum_{i} m_{i} Y_{i}
$$

where the summation is taken over all the irreducible components of $Y_{\text {red. }}$. Since $Q_{i}$ is unique for such $Y_{i},[Y]$ is well-defined. We call [Y] the compact $q$-cycle associated with $Y$. Note that the notation is compatible with that of Lemma 1.3.

The following lemma which we need later is found in [3, Théorème 1, p.38] up to a slight improvement.

Lemma 3. 1. 1) Let $\left\{A_{s} ; s \in S\right\}$ be an analytic family of compact q-cycles of a complex space $X$ parametrized by a reduced complex space $S$. Then the set $W$ defined by $W=\left\{(s, x) ; x \in\left|A_{s}\right|\right\}$ is an analytic subset of $S \times X$ and the natural projection $f: W \rightarrow S$ is proper. 
Let $W=\cup_{j} W_{j}$ be the decomposition of $W$ into irreducible components, and put $f_{j}=\left.f\right|_{w_{j}}$. Then for every $j$ there exists an integer $n_{j}$ and $a$ dense Zariski open subset $V$ of $S$ such that for every $s \in V, A_{s}$ is given by $A_{s}=\sum_{j} n_{j}\left[W_{j s}\right]$, where we regard $W_{j s}$ naturally as a subspace of $X \cong\{s\} \times X$.

2) Conversely, let $S$ be a reduced complex space and $W$ an analytic subset of $S \times X$. Let $f: W \rightarrow S, W=\cup W_{j}$ and $f_{j}: W_{j} \rightarrow S$ be as in 1). Suppose that $f$ is proper, each fiber of $f$ has pure dimension $q$ and all the $f_{j}$ are surjective. Then if $S$ is weakly normal, then for every $k$-tuple of positive integers $\left(n_{1}, \cdots, n_{k}\right)$ there exists an analytic family, $\left\{A_{s}\right\}$, of compact q-cycles of $X$ parametrized by $S$ such that for some dense Zariski open subset $V$ of $S, A_{s}=\sum n_{j}\left[W_{j s}\right]$ for $s \in V$.

Proof. In view of the proof of [3, Théorème 1] we have only to show the existense of $V$ as stated in 1 ) of the above Lemma. We shall take as $V$ any dense Zariski open subset of $S$ such that $W_{s}$ is generically reduced. Such a $V$ exists by Lemma 1.2. By Frisch [6] we may further assume that $f$ is flat over $V$. Now since $\left|A_{s}\right|=W_{s, \text { red, }}$ we may write $A_{s}=\sum_{\alpha=1}^{N_{s}} n_{s \alpha} W_{s \alpha}$ for some $n_{s \alpha}>0$, where $W_{s \alpha}$ are irreducible components of $W_{s, \text { red. }}$. For any $s \in V$ and any $W_{s \alpha}$ take a nonsingular point $w \in W_{s a}$. Then since $f$ is flat at $w, f$ is smooth at that point. Let $U$ be the dense Zariski open subset of $X$ defined by $U=\left\{x \in f^{-1}(V) ; f\right.$ is smooth at $\left.x\right\}$. For any point $x \in U$ let $W_{f(x) \alpha}$ the unique irreducible component of $W_{f(x)}$ passing through $x$ and $n_{x}=n_{f(x) \alpha}$. Then by the definition of analytic family it is obvious that $n_{x}$ is locally constant on $U$. Hence it is constant, say $n_{j}$, on $U \cap W_{j}$ for every $j$. Since for $s \in V, W_{s \alpha} \cap U \neq \varnothing$ for all $\alpha$ as we have seen above, this proves the lemma.

We call $W \subseteq S \times X$ and the morphism $f: W \rightarrow S$ in 1) associated with the analytic family $\left\{A_{s}\right\}$.

3. 2. Let $f: X \rightarrow S$ be a morphism of complex spaces. For every reduced complex space $T$ over $S$ with the structure morphism $\alpha: T \rightarrow S$ we mean by an analytic family of compact $q$-cycles of $X / S$ parametrized by $T$ an analytic family of compact $q$-cycles, $\left\{A_{t}\right\}$, of $X$ parametrized by $T$ such that for all $t \in T f\left(\left|A_{t}\right|\right) \leqq \alpha(t)$. Let $\left(\mathcal{A n}_{n_{\text {red }}} / S\right)$ be the category of re- 
duced complex spaces over $S$ and $S$-morphisms. Let $F_{S, q}:\left(\mathcal{A} n_{\text {red }} / S\right)$ $\rightarrow$ (Sets) be the contravariant functor defined by $F_{S, q}(T)=$ the set of analytic families of compact $q$-cycles $X / S$ parametrized by $T$. Starting from the Barlet's rcsult in 2.2 we easily show the following.

Proposition 3.2. For cvery $q \geqq 0, F_{S . q}$ is representable in $\left(\ln n_{\mathrm{red}} / S\right)$

Proof. We first make the following general remark. Let $g: X \rightarrow Y$ and $h: Y \rightarrow Z$ be proper morphisms of reduced and irreducible complex spaces. Suppose that every fiber of $h g$ has pure dimension $q \geqq 0$ and that $g$ is surjective. Then every fiber of $h$ has pure dimension $q^{\prime}$, where $q^{\prime}$ $=\operatorname{dim} Y-\operatorname{dim} Z$. In fact this follows easily from the upper semicontinuity of the dimension of the fibers of $g$ and $h$, shown by Remmert.

Now let $B_{g}(X)$ be the Barlet space of compact $q$-cycles of $X$ and $\left\{A_{b} ; b \in B_{q}(X)\right\}$ be the universal family of compact $q$-cycles parametrized by $B_{q}(X)$. Let $W \subseteq B_{q}(X) \times X$ be the associated analytic subset and $\tilde{\pi}: W \rightarrow B_{q}(X)$ the natural projection. Put $\bar{f}=i d \times f: B_{q}(X) \times X \rightarrow B_{q}(X)$ $\times S$. Let $\bar{W}=\bar{f}(W) \subseteq B_{q}(X) \times S$ and $\pi: \bar{W} \rightarrow B_{q}(X)$ the natural projection. Let $B^{\prime}$ be the union of those irreducible components, $B_{\alpha}$, of $B_{q}(X)$ for which the induced morphism $\bar{W}_{B_{\alpha}} \rightarrow B_{\alpha}$ has relative dimension zero i.e. $\operatorname{dim} \bar{W}_{B_{\alpha}}=\operatorname{dim} B_{\alpha}$ since the map is surjective. Let $\bar{W}^{\prime}=\bar{W}_{B^{\prime}}$ and $\pi^{\prime}=\left.\pi\right|_{\bar{W}}$. We show that $\pi^{\prime}$ is a finite morphism. It is enough to see that $\operatorname{dim} \pi^{\prime-1}(b)=0$ for every $b \in B^{\prime} . \quad$ Let $\bar{W}_{i}$ be any irreducible component of $\bar{W}$ and $W_{i}$ any irreducible component of $W$ which is mapped onto $\bar{W}_{i}$ by $\bar{f}$. Let $\bar{f}_{i}=\left.\bar{f}\right|_{w_{i}}$ and $\pi_{i}^{\prime}=\left.\pi^{\prime}\right|_{\bar{w}_{i}}$. Then every fiber of $\bar{f}_{i}$ has pure dimension $q$. Hence we can apply the above remark to the morphisms $\bar{f}_{i}: W_{i} \rightarrow \bar{W}_{i}$ and $\pi^{\prime}: \bar{W}_{i} \rightarrow B^{\prime}$ to see that every fiber of $\pi_{i}^{\prime}$ and hence of $\pi^{\prime}$ has pure dimension zero as desired.

Let $\nu: \widetilde{B} \rightarrow B^{\prime}$ be the normalization of $B^{\prime}$. For every irreducible component, $\widetilde{B}_{\alpha}$, of $\widetilde{B}$ let $k=k_{\alpha}$ be the degree of the induced finite morphism $\tilde{\pi}_{\alpha}: \bar{W}_{B_{\alpha}} \rightarrow B_{\alpha}$. Let $\rho_{k}: S \times \cdots \times S$ (k-times) $\rightarrow \operatorname{sym}^{k} S$ be the natural projection. Then we can define as in 2.2 (cf. also $[3, \mathrm{p} .25]$ ) a morphism $\varphi_{\alpha}: B_{\alpha} \rightarrow \operatorname{sym}^{k} S$ such that (*) $\bar{W}_{B_{c}}=\operatorname{supp} \varphi_{c c}=\left\{(b, s) \in B_{\alpha} \times S ; s=s_{1}\right.$ for some $\left.\left(s_{1}, \cdots, s_{k}\right) \in \rho_{k}^{-1}\left(\varphi_{\alpha}(u)\right)\right\}$. Let $J_{k}$ be the diagonal in $S \times \cdots \times S$ i.e. 
$\Delta_{k}=\{(s, \cdots, s) ; s \in S\}$ and $\bar{\Delta}_{k}=\rho_{k}\left(\Delta_{k}\right)$. Then define $C_{\alpha}=\nu\left(\varphi_{\alpha}^{-1}\left(\bar{J}_{k}\right)\right)$ with reduced structure and $C=\cup_{\alpha} C_{\alpha}$. By $\left(^{*}\right)$ and the definition of $B^{\prime}$ it follows easily that $C$ coinsides with the set $C^{\prime}=\left\{b \in B_{q}(X) ; \pi^{-1}(b)\right.$ consists of a single point $\}$.

Now we set $B_{0}=\bar{W}_{c \text {,red. }}$ On $B_{0}$ we have the natural analytic family $\left\{A_{b}^{\prime} ; b \in B_{0}\right\}$ of compact $q$-cycles of $X / S$ defined by $A_{b}^{\prime}=A_{\pi(b)}$. We show the universality of this family. Let $\left\{A_{t} ; t \in T\right\}$ be any analytic family of compact $q$-cycles of $X / S$ parametrized by a reduced complex space $T$ over $S$. Then by the universality of $B_{q}(X)$ we have the unique morphism $h: T \rightarrow B_{q}(X)$ such that $A_{t}=A_{h(t)}$. Let $\alpha: T \rightarrow S$ be the structure morphism. Then since $f\left(\left|A_{t}\right|\right)=\alpha(t)$, by the above characterization of $C$ we see that $h$ factors through $C$. (Note that $\left|A_{h(t)}\right|=W_{h(t), \text { red.) }}$ Thus if we put $\tilde{h}=h \times \alpha: T \rightarrow C \times S$, then we see readily that $\tilde{h}(T) \subseteq B_{0}$. Moreover it is clear that $A_{t}=A_{h(t)}^{\prime}$ for all $t \in T$. Finally by definition $B_{0}$ is reduced and has the natural projection to $S$ as a subspace of $C \times S$ with respect to which $\tilde{h}$ is an $S$-morphism. This shows the universality of our family $\left\{A_{b}^{\prime} ; b \in B_{0}\right\}$.

Q.E.D.

We denote the space $B_{0}$ obtained in the above proof which represent the functor $F_{S, q}$ by $B_{q}(X / S)$ and call it the relative Barlet space associated with $X / S$. As the above proof shows we have the natural projection $j: B_{q}(X / S) \rightarrow B_{q}(X)$ which is a homeomorphism onto the subspace $C$.

3. 3. Let $S$ be a complex space and $X$ a complex space over $S$. We recall the definition of the Douady space, $D=D_{X / S}$, of $X$ over $S$. First let $T$ be a complex space over $S$. Then a flat family of a compact subspaces of $X$ parametrized by $T$ over $S$ is a subspace $Y$ of $X_{T}$ which is proper and flat over $T$ via the natural projection $Y \rightarrow T$. Let $\left(A_{n} / S\right)$ be the category of complex spaces over $S$ and $S$-morphisms. Let $F$ : ( $A_{n}$ $/ S) \rightarrow$ (Sets) be the contravariant functor defined by $F(T)=$ the set of flat families of compact subspaces of $X$ parametrized by $T$ over $S$. Then Douady in [5] showed that $F$ is representable, the generalization to the relative case being due to Pourcin [17]. Namely there exists a complex space $D$ over $S$ and a subspace $Z$ of $X_{D}$ which is proper and flat over $D$ such that for every $Y \subseteq X_{T}$ as above there exists a unique $S$-morphism 
$h_{2}: T \rightarrow D$ with $Y=Z_{T}:=Z \times_{D} T$, where $T$ is over $D$ by $h$. In particular the points of $D_{\text {red }}$ correspond bijectively to the set of all compact subspaces of $X$. We put $D=D_{X / S}$ (resp. $Z=Z_{X / S}$ ) and call it the Douady space of $X$ orer $S$ (resp. the universal family associated with $X / S$ ). If $S$ reduces to a point, then we write $D_{X}$ (resp. $Z_{X}$ ) instead of $D_{X / S}$ (resp. $\left.Z_{X / S}\right)$. Then we have the natural embedding $j: D_{X / S} \rightarrow D_{X}[17]$.

For every integer $q$ we define

$$
D_{X / S, q}=\left\{d \in D_{X / S, \text { red }} ; Z_{d} \text { has pure dimension } q\right\} .
$$

Then by the flatness of $Z$ over $D_{X / S}, D_{X / S, q}$ is the union of irreducible components of $D_{X / S}$. Now to every $\mathrm{d} \in D_{X / S, q}$ we associate a compact $q$-cycle $\rho_{0}(d)$ of $X$ by $\rho_{0}(d)=\left[Z_{d}\right]$ (cf. 3.1), considering $Z_{d}$ naturally as a subspace of $X$. We may then regard $\rho_{0}$ as a map $\rho_{0}: D_{X / S, q} \rightarrow B_{q}(X)$. Define $\rho^{\prime}: D_{X / S, q} \rightarrow B_{q}(X) \times S$ by $\rho^{\prime}(d)=\left(\rho_{0}(d), \pi(d)\right)$, where $\pi: D_{X / S} \rightarrow S$ is the natural projection. Then we see immediately that $\rho^{\prime}$ factors through $B_{q}(X / S)$. The resulting map

$$
\rho_{q}=\rho_{X / S, q}: D_{X / S, q} \rightarrow B_{q}(X)
$$

is called the Barlet map associated with $X / S$. In fact Barlet proved in $[3$, Théorème 8$]$ the following

Theorem (Barlet). For every $q \geqq 0, \rho_{q}$ is a morphism of complex spaces.

Combining this theorem with Proposition 2.3 we get easily the following

Corollary 3. 3. Let $f: X \rightarrow S$ be a proper and flat morphism of reduced complex spaces. Suppose that every fiber of $f$ has pure dimension $q \geqq 0$. Then for every continuous $2 q$-form, $\chi$, on $S$ the function $\lambda(s)=\int_{\left[X_{s}\right]} \chi$ is continuous on $S$.

Remark 3.1. More precisely one can directly show the following. Let $f: X \rightarrow S$ be a proper morphism of reduced complex spaces. Suppose that every fiber of $f$ has pure dimension $q$ and that every irreducible component of $X$ is mapped surjectively onto $S$. Then the following con- 
ditions are equivalent. 1) For every continuous $2 q$-form $\chi$ on $X$ the function $\lambda(s)=\int_{\left[X_{s}\right]}$ is continuous. 2) For every $s \in S$ there is a dense Zariski open subset $U_{s}$ of $X_{s}$ such $f$ is flat at every point of $U_{s}$. (For related results see Stoll [23, Theorem 3.8] and King [15, Theorem 3. 3. 2].)

3. 4. For every $q \geqq 0$ define the subspace, $\bar{D}_{q}=\bar{D}_{X / S, q}$, of $D_{X / S, q}$ by $\bar{D}_{q}$ $=\cup D_{\alpha}$, where $D_{\alpha}$ are those irreducible components of $D_{X / S, q}$ for which the induced map $Z_{D_{\alpha}} \rightarrow D_{\alpha}, Z=Z_{X / S}$, satisfies the equivalent conditions of Lemma 1. 4. We set $\bar{D}_{X / S}=\coprod_{q} \bar{D}_{X / S, q}$. For simplicity, here we introduce the following terminology.

We say that $X$ has the property $B_{q} P\left(\right.$ resp. $\bar{D}_{q} P$ ) over $S$ if every irreducible component of $B_{q}(X / S)$ (resp. $\bar{D}_{X / S, q}$ ) is proper over $S$. If $X$ has the property $B_{q} P$ for all $q \geqq 0$, then we say that it has the property $B P$ over $S$. Further if $S$ is a point, we omit 'over $S$ '.

Proposition 3.4. Suppose that $X$ has the property $B_{q} P$ over $S$. Let $\bar{\rho}_{q}: \bar{D}_{X / S, q} \rightarrow B_{q}(X)$ be the restriction of the Barlet map to $\bar{D}_{X / S, q}$. Then $\bar{\rho}_{q}$ is proper. In particular $X$ has the property $\bar{D}_{q} P$ over $S$.

Proof. Let $B_{0}$ be any irreducible component of $B_{q}(X / S)$ which intersects with the image of $\bar{D}=\bar{D}_{X / S, q}$. Let $\left\{A_{b}, b \in B_{0}\right\}$ be the corresponding analytic family of compact $q$-cycles of $X$ over $S$. We define inductively a descending sequence, $B_{0} \supseteqq B_{1} \supseteq \cdots \supseteqq B_{k} \cdots$, of reduced subspaces of $B_{0}$ by the following requirement; let $f_{i}: W_{i} \rightarrow B_{i}$ be the morphism associated with the family $\left\{A_{b} ; b \in B_{i}\right\}$ (cf. Lemma 3.1), and $U_{i}$ the dense Zariski open subset of $W_{i}$ defined by $U_{i}=\left\{b \in B_{i} ; W_{i b}\right.$ is reduced and $f$ is flat at every point of $\left.W_{i b}\right\}$ (Lemma 1.5). Then $B_{i+1}=B_{i}-U_{i}$. Now let $D_{\alpha}$ be any irreducible component of $\bar{D}$ such that $\bar{\rho}_{q}\left(D_{\alpha}\right) \subseteq B_{0}$, and $V_{\alpha}=\left\{d \in D_{\alpha} ; Z_{d}\right.$ is reduced $\}$, where $Z=Z_{X / S}$. Note that $\bar{\rho}_{q}$ is injective on $V_{\alpha}$ by the definition of the Barlet map. Let $m$ be the minimal index such that $\bar{\rho}_{q}\left(V_{\alpha}\right) \varsubsetneqq B_{m+1}$. Then $\bar{\rho}_{q}\left(V_{\alpha}\right)$, and hence $\bar{\rho}_{q}\left(D_{\alpha}\right)$, is contained in $B_{m}$. Take any irreducible component $B_{m \alpha}$ of $B_{m}$ which contains $\bar{\rho}_{q}\left(D_{\alpha}\right)$. Let $W_{m \alpha}=W_{B_{m \alpha}}$ and $f_{m \alpha}: W_{m \alpha} \rightarrow B_{m \alpha}$ be the induced map. Take a flattening $\tilde{f}_{m \alpha}: \widetilde{W}_{m \alpha} \rightarrow \widetilde{B}_{m \alpha}$ of $f_{m \alpha}$ with the flattening morphism $\phi: \widetilde{B}_{m \alpha}$ 
$\rightarrow B_{m \alpha}$ (cf. 1.4). Then since $\widetilde{W}_{m \alpha}$ is naturally the subspace of $X_{\widetilde{B}_{m \alpha}}$ $=X \times{ }_{S} \widetilde{B}_{m \alpha}$, by the universality of $D_{X / S}$ we have the unique $S$-morphism $\tau: \widetilde{B}_{m a} \rightarrow D_{X / S}$ such that $W_{m \alpha}=Z_{\widetilde{B}_{m \alpha}}:=Z \times_{D_{X / S}} \widetilde{B}_{m \alpha}$, where $\widetilde{B}_{m \alpha}$ is over $D_{X / S}$ by $\tau$. On the other hand, note that since $f_{m \alpha \alpha}$ is flat on $U_{m \alpha}=B_{m \alpha}-B_{m+1}$ by our construction, $\phi$ gives as isomorphism of $\phi^{-1}\left(U_{m \alpha}\right)$ and $U_{m \alpha}$. Let $d \in V_{\alpha}$ be any point with $\bar{d}=\bar{\rho}_{q}(d) \in U_{m \alpha}$. Then since $W_{m \alpha, d}$ is reduced by our choice of $U_{m}$, we have $Z_{d}=W_{m \alpha, d}$ considering both sides naturally as a subspace of $X$. Hence $\tau \cdot \phi^{-1}(\bar{d})=d$. This implies that $\tau\left(\widetilde{B}_{m \alpha}\right) \cap V_{\alpha}$ $\supseteqq \bar{\rho}_{q}^{-1}\left(U_{m \alpha}\right) \cap V_{\alpha}$ which is a nonempty Zariski open subset of $D_{\alpha}$ by our choice of $m$. It follows that $\tau\left(\widetilde{B}_{m \alpha}\right) \leqq D_{\alpha}$, since $\widetilde{B}_{m \alpha}$ is irreducible. Finally since $\widetilde{B}_{m \alpha}$ is proper over $S$ as well as $B_{m \alpha}$ and $\tau$ is an $S$-morphism, $\tau$ must be surjective. Hence $D_{\alpha}$ also is proper over $S$. Moreover as is clear from the above proof we have the following commutative diagram of proper bimeromorphic $S$-morphisms

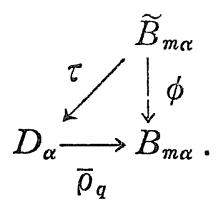

Let $D_{\beta}$ be another irreducible component of $\bar{D}$ with $\bar{\rho}_{q}\left(D_{\beta}\right) \cap B_{0} \neq \varnothing$. Then take $m^{\prime}$ and an irreducible component $B_{m^{\prime} \beta}$ of $B_{m^{\prime}}$ as for $D_{\alpha}$. Then since $\bar{\rho}_{q}$ gives a bimeromorphic morphism of $D_{\beta}$ to $B_{m^{\prime} \beta}$ we infer readily that $B_{m^{\prime} \beta} \neq B_{m \alpha}$. Now let $K$ be any compact subset of $B_{q}(X / S)$. Then there are only finitely many irreducible components of $B_{q}(X / S)$ which intersects with $K$. For each such irreducible component, say $B_{0}$, there are only finitely many $B_{m}$ as above with $B_{m} \cap K \neq \phi$. And finally for each such $m$ there are finitely many irreducible components of $B_{m}$ intersecting with $K$. From this combined with above remark it follows then easily that $\bar{\rho}_{q}^{-1}(K)$ is compact in $\bar{D}$. This shows the properness of $\bar{\rho}_{q}$.

Q.E.D.

We can drop the pure dimensional assumption in the above proposition (cf. the remark below).

Lemma 3. 5. Let $f: X \rightarrow S$ be a proper morphism of complex spaces of relative dimension $q \geqq 0$. Let $D_{\alpha}$ be any irreducible com- 
ponent of $D_{X / s, \text { red }}$ such that $Z_{\alpha}=Z_{D_{\alpha}}$ is reduced. Then there is an analytic subset $Y$ of $\bar{D}_{X / S}^{(q)}=\bar{D}_{X / S, q} \times_{S} \cdots \times_{S} \bar{D}_{X / S, 0}$ and a generically surjective meromorphic map $\tau: Y \rightarrow D_{\alpha}$ (i.e. $\tau(Y)$ contains a dense Zariski open subset of $D_{a}$ ).

Proof. Let $Z_{\alpha, i}, 0 \leqq i \leqq q$, be the union of all the irreducible components of relative dimension $i$ of $Z_{\alpha}$. By Frisch [6] there is a dense Zariski open subset $U$ of $D_{\alpha}$ such that $Z_{\alpha, i}$ are all flat over $U$. Then by the universality of $D_{X / S}$ we have a unique $S$-morphism $h_{i}: U \rightarrow D_{X / S, i}$ such that $\left(Z_{\alpha, i}\right)=\left(Z_{X / S}\right)_{U}$. Since $Z_{i}$ are reduced, the image of $h_{i}$ are in fact contained in $\bar{D}_{X / S}$. Define $h=h_{q} \times{ }_{s} \cdots \times{ }_{S} h_{0}: U \rightarrow \bar{D}_{X / S}^{(q)}$. Let $A$ be the minimal analytic subset containing $h(U)$.

On the other hand, let $Z_{(q), i}$ be the pull back of $Z_{X / S, i}$ to $\bar{D}_{X / S}^{(q)}$ via the natural projection $\bar{D}_{X / S}^{(q)} \rightarrow \bar{D}_{X / S, i}$. Put $Z^{(q)}=\bigcup_{i} Z_{i}^{(q)}$. Then again by Frisch there is a dense Zariski open subset $W$ of $A$ such that $Z_{A}^{(q)}$ is flat over $W$. By the universality of $D_{X / S}$ we have a unique $S$-morphism $\tau: W \rightarrow D_{X / S}$ such that $Z_{W}^{(q)}=\left(Z_{X / S}\right)_{W}$. By the minimality of $A, U \cap h^{-1}(W)$ $\neq \varnothing$, and by the construction we see readily that $\tau h=$ identity on $U_{1}$ $=U \cap h^{-1}(W)$. In particular $\tau(W) \subseteq D_{a}$ and it contains a Zariski open subset of $D_{\alpha}$. Finally that $\tau$ extends to a meromorphic map from $A$ follows from Lemma 5. 1 below.

Q.E.D.

Remark 3.2. Lemma 5.1 implies more generally that if $X$ has property $\bar{D}_{q} P$ over $S$, then $Z_{\alpha}$ in the above lemma is proper over $D_{\alpha}$.

\section{§4. Closedness of Barlet Space in the Kähler Case}

4. 1. We begin with the following proposition.

Proposition 4. 1. Let $S$ be a complex space and $X$ a complex space over $S$. Suppose that there exists a closed positive $C^{\infty}$ form $\Omega$ of type $(q, q)$ on $X$ with $q>0$ (cf. Def. 1.2.). Then every connected component of $B_{q}(X / S)$ is locally bounded in the sense that for every connected component, say $B_{0}$, of $B_{q}(X / S)$ a subset $E$ of $B_{0}$ is bounded if $\cup\left|A_{b \in E}\right|$ is contained in a compact subset of $X$. 
Proof. Let $B_{0}$ be any connected component of $B_{q}(X / S)$, and $\left\{A_{b}\right.$; $\left.b \in B_{0}\right\}$ be the associated family of compact $q$-cycles of $X$ parametrized by $B_{0}$. Then by Lemma 1.1 it is enough to show that the function $\lambda(b)=\int_{A_{b}} \Omega$ is bounded on $B_{0}$. Since $\lambda$ is continuous on $B_{0}$ by Proposition 2.3 , to prove this it is enough to show that for every irreducible component of $B_{0}$ it is constant. Let $B_{1}$ be any irreducible component of $B_{0}$. Then again by continuity we have only to show that $\lambda$ is constant on some Zariski open subset of $B_{1}$. Now by Lemma 3. 1 the set $W=\{(b, x)$; $x \in\left\{A_{b} \mid\right\}$ is an analytic subset of $B_{1} \times X$. Let $\pi: W \rightarrow X$ and $f: W \rightarrow B_{1}$ be the natural projections. Let $W_{j}, 1 \leqq j \leqq s$, be the irreducible components of $W$. Then by Lemma 3.1 there exists a dense Zariski open subset $V_{1}$ of $B_{1}$ and a positive integer $n_{j}$ for each $j$ such that $A_{b}=\sum n_{j}\left[W_{j b}\right]$ for every $b \in V_{1}$. By Lemma 1.5 restricting $V_{1}$ if necessary we may assume that $W_{b}$ is reduced for every $b \in V_{1}$. Finally put $V=V_{1} \cap B_{1}{ }^{*}$, where $B_{1}^{*}$ is the set of nonsingular points of $B_{1}$. We show that $\lambda$ is constant on $V$. Let $b_{1}$ and $b_{2}$ be any two points of $V$. Then we connect $b_{1}$ and $b_{2}$ by a peicewise real analytic curve in $V$. Namely we take a finite number of points $a_{1}, \cdots, a_{k}$ of $V$ with $a_{1}=b_{1}$ and $a_{k}=b_{2}$ and real analytic embeddings $h_{i}: I \rightarrow V, 1 \leqq i \leqq k-1$, with $h_{i}(0)=a_{i}$ and $h_{i}(1)=a_{i+1}$, where $I$ is the interval $[0,1]$. Here a map $h: I \rightarrow V$ is called a real analytic embedding if for some $\varepsilon>0 h$ extends to a real analytic embedding of $(-\varepsilon, 1+\varepsilon)$ into an open subset of $V$. We then show that $\left.{ }^{*}\right) \lambda\left(a_{i}\right)$ $=\lambda\left(a_{i+1}\right)$ for all $i$. Let $h_{i}(I)=I_{i}$, and $f_{j}=\left.f\right|_{W_{j} \text {. }}$ Then $\widetilde{I}_{i j}=f_{j}^{-1}\left(I_{i}\right)$ is obviously a semianalytic set in $W$ with boundary $f_{j}^{-1}\left(a_{i}\right) \cup f_{j}^{-1}\left(a_{i+1}\right)$. Give a suitable orientation on $\widetilde{I}_{i j}$. Then we consider the semianalytic chain $C_{i}=\sum_{j} n_{j} \widetilde{I}_{i j}$ on $W$ whose boundary as a semianalytic chain is given by $\pm \partial C_{i}=\sum_{j} n_{j} f_{j}^{-1}\left(a_{i}\right)-\sum_{j} n_{j} f_{j}^{-1}\left(a_{i+1}\right)=\sum_{j} n_{j}\left[W_{j a_{j}}\right]-\sum_{j} n_{j}\left[W_{j a_{i+1}}\right]=A_{a_{i}}-A_{a_{i+1}}$. Here the third equality comes from the fact that $W_{j a_{k}}$ are all reduced. Now let $\widetilde{\Omega}=\pi^{*} \Omega$. Then by Stokes formula on semianalytic sets (cf. 1.2) we have the equality $0=\int_{C_{i}} d \widetilde{\Omega}=\int_{\partial C_{i}} \widetilde{\Omega}=\int_{A a_{i}} \Omega-\int_{A a_{i+1}} \Omega$. This shows $*)$. It follows that $\lambda\left(b_{1}\right)=\lambda\left(b_{2}\right)$ as desired.

Q.E.D.

Let $S$ be a complex space and $X$ a complex space over $S$. For every integer $k>0$ let $\operatorname{sym}_{S}^{k} X$ be the $k$-th symmetric product of $X$ over $S$. Namely it is the quotient analytic space of $X \times{ }_{s} \cdots \times{ }_{s} X$ (k-times) by the 
natural action on it of the symmetric group $S_{k}$ of degree $k$. We shall omit the proof of the following lemma which one proves easily according to the definitions.

Lemma 4. 2. There is a natural isomorphism $B_{0}(X / S) \cong \prod_{k \geqq 1}$ $\operatorname{sym}_{S}^{k} X$.

Theorem 4. 3. Let $S$ be a complex space and $X$ a complex space proper over $S$. Suppose that there exists a closed positive $C^{\infty}$ form, $\Omega$, of type $(q, q)$ on $X$. Then every connected component of $B_{q}(X / S)$ (resp. $\bar{D}_{X / \mathrm{S}, q}$ ) is proper over $S$.

Proof. Let $B$ be any connected component of $B_{q}(X / S)$ and $\alpha: B$ $\rightarrow S$ (resp. $\pi: X \rightarrow S$ ) the natural map. Let $K$ be any compact subset of $S$. We show that $\alpha^{-1}(K)$ is compact. Suppose first that $q=0$. Then by Lemma $4.2 \alpha^{-1}(K)$ is isomorphic to a subspace of $\operatorname{sym}_{\mathbb{K}}^{k} \pi^{-1}(K)$ for some $k>0$ and hence is compact. Next suppose that $q>0$. Let $\left\{A_{b}\right.$; $\left.b \in B_{q}(X / S)\right\}$ be the universal family. Then $\underset{b \in \alpha^{-1}(K)}{\cup}\left|A_{b}\right|$ is contained in the compact set $\pi^{-1}(K)$. Hence by Proposition $4.1 \alpha^{-1}(K)$ is bounded and then by Proposition 2.10 it is relatively compact and hence compact in $B_{q}(X / S)$. Finally the properness of $\bar{D}_{X / S}$ follows from Proposition 3. 4.

Remark 4.1. The above theorem answers a certain question asked by $\mathrm{Siu}$ in $[21,3.3]$.

4. 2. We relativize the definition of a Kähler space as follows.

Definition 4. 1. Let $f: X \rightarrow Y$ be a morphism. We call $f$ a Kähler morphism, if there exists an open covering $\mathfrak{U}=\left\{U_{\alpha}\right\}$ of $X$ and a system of $C^{\infty}$ functions, $\left\{p_{\alpha}\right\}$, each defined on $U_{\alpha}$ such that for every $y \in Y 1$ ) $p_{\alpha}$ is strictly plurisubharmonic when it is restricted on $U_{\alpha} \cap X_{y}$ and similarly $p_{\alpha}-p_{\beta}$ is pluriharmonic on $U_{\alpha} \cap U_{\beta} \cap X_{y}$.

Remark 4.2. 1) Note that if $f$ is Kähler and $p_{\alpha}$ are as above, 
then $\left\{\sqrt{-1} \partial \bar{\partial} p_{\alpha}\right\}$ give a real closed $(1,1)$-form $\omega$ globally defined on $X$. We call such an $\omega$ a relative Kähler form for $f$. If $f$ is a constant map, then $\omega$ is a Kähler form on $X$ and $X$ is a Kähler space in the sense of Definition 1.2.

2) $\mathrm{By}$ definition it is clear that every subspace of a Kähler space is again Kähler. In particular every projective variety is Kähler. On the other hand, we remark that if $X$ is not projective, then $X$ can be nonKähler even if $X_{\text {red }}$ is Kähler. An example can be given using an example of Moisezon [16, §2].

In the following lemma we give some of the most elementary facts on Kähler morphisms.

Lemma 4. 4. 1) Let $f: X \rightarrow Y$ be a projective morphism. Then $f$ is Kähler.

2) Let $f: X \rightarrow Y$ be a proper Kähler morphism. Suppose that $Y$ is Kähler. Then for every relatively compact subdomain $G$ of $Y, f^{-1}(G)$ is Kähler.

Proof. 1) follows directly from the definitions and the details will be omitted (cf. the proof of [6, Lemma 2]). We show 2). Let $\omega_{X}$ be a relative Kähler form on $X$ and $\omega_{Y}$ a Kähler form on $Y$. Then we infer readily that there exists a positive constant $M$ such that $\omega_{X}+M f^{*}\left(\omega_{Y}\right.$ is a Kähler form on $f^{-1}(G)$ (cf. the proof of [6, Lemma 2]). Q.E.D.

Theorem 4. 5. Let $f: X \rightarrow S$ be a proper Kähler morphism. Then every connected component of the relative Barlet space $B(X / S)$ (resp. $\left.\bar{D}_{X / S}\right)$ is proper over $S$.

Proof. Let $B$ be any connected component of $B(X / S)$ and $\alpha: B$ $\rightarrow S$ (resp. $\pi: X \rightarrow S$ ) the natural projection. Let $U$ be any relatively compact Stein open subset of $S$ and hence is a Kähler space. Since the problem is local on $S$, it is enough to show that $\left.\alpha\right|_{\alpha^{-1}(U)}$ is proper. First by Lemma 4.42$) \pi^{-1}(U)$ is Kähler. Let $\omega$ be a Kähler form on $\pi^{-1}(U)$. Then $\omega^{q}$ is a closed positive $C^{\infty}$ form of type $(q, q)$ on $\pi^{-1}(U)$. Then almost as in the proof of Theorem 4.3 we can show that for 
Remark 4.3. There are examples of compact non-Kähler manifolds with non-compact irreducible components for $\bar{D}_{X}$, and hence also for $B(X)$ by Proposition 3.4. Here we shall give one of them. For another example see $[8,4.7]$.

We use an example of an algebraic surface, $S$, found in $[19, \mathrm{p} .164]$. $S$ is a rational elliptic surface over a complex projective line $\mathbb{P}^{1}$ which is obtained by blowing up nine general points on the complex projective plane $\mathbb{P}^{2}$, and with a fiber preserving automorphism of infinite order, $g$. In fact $g$ restricted to general fibers of the elliptic fibering of $S$ gives translations of infinite order on them. Moreover the exceptional curves of the first kind, $E_{i}, i=1, \cdots, 9$, obtained by the blowing up are sections to this fibering i.e. mapped biholomorphically onto $\mathbb{P}^{1}$. Now let $C$ be a nonsingular elliptic curve with a fixed system of generators, $\{\alpha, \beta\}$, for the fundamental group $\pi_{1}(C)$. Define the homomorphism $\rho$ $: \pi_{1}(C) \rightarrow$ AutS by $\rho(\alpha)=g$ and $\rho(\beta)=e$. Then corresponding to $\rho$ we have in a usual manner a fiber bundle $f: X \rightarrow C$ with typical fiber $S$ with constant transition functions. Take a point $u \in \mathbb{P}^{1}$ and identify $S$ with the fiber $X_{u}$. Let $E$ be one of the $E_{i}$. Let $D_{\alpha}$ be the irreducible component of $\bar{D}_{X}$ containing the point corresponding to the subspace $E \subseteq S \subseteq X$. We show that $D_{\alpha}$ is non-compact. Assume the contrary. Let $Z_{\alpha}=\left(Z_{X}\right)_{D_{\alpha}}$ be the universal family restricted to $D_{\alpha}$ and $\pi_{\alpha}: Z_{\alpha} \rightarrow X$ the natural projection. Since by our assumption $Z_{\alpha}$ is compact, $T=\pi_{\alpha}\left(Z_{\alpha}\right)$ is an analytic subset of $X$ by a theorem of Remmert. On the other hand, by our construction we see readily that $X_{u} \cap T=\cup_{m \in Z} g^{m}(E)$, where $g^{m}(E)$ is the image of $E$ by the automorphism $g^{m}$ on $X_{u}=S$. Hence $\cup g^{m}(E)$ must be an analytic subset of $S$, which is a contradiction since $g^{m}(E) \cap F$ consists of infinite number of discrete points of $F$ by what we have said above, where $F$ is a general fiber of the elliptic fibering of $S$. In particular $X$ is not Kähler, though it is not difficult to verify this directly.

4. 3. We now generalize Theorem 4.4 in the absolute case to a more general class of complex spaces, namely, that of reduced compact complex spaces which are meromorphic images of compact Kähler spaces. We sım- 
marize some elementary properties of this class, say $\mathscr{C}$, in the following lemma.

Lemma 4. 6. Let $X$ be a reduced compact complex space and $Y \in \mathscr{C}$. Then

1) $X \in \mathscr{C}$ if and only if it is a holomorphic image of a compact Kähler manifold.

2) If $X$ is isomorphic to a subspace of $Y$, then $X \in \mathscr{C}$.

3) If $X$ is a meromorphic image of $Y$, then $X \subseteq \mathscr{C}$.

4) If there exists a Kähler morphism $f: X \rightarrow Y$, then $X \in \mathscr{C}$.

Proof. 1) Let $X \in \mathscr{C}$. By definition there exists a surjective meromorphic map $f: \widetilde{X} \rightarrow X$, where $\widetilde{X}$ is a reduced compact Kähler space. Then by Hironaka [14] there exists a projective bimeromorphic morphism $g: X_{1} \rightarrow \widetilde{X}$ such that $f g$ is a morphism. In fact let $X^{\prime}$ be the graph o $f$ in $\widetilde{X} \times X$ and $p: X^{\prime} \rightarrow \widetilde{X}$ the natural projection. Then by $[14$, Cor. 2] there is a projective bimeromorphic morphism $g: X_{1} \rightarrow \widetilde{X}$ such that $p^{-1} g: X_{1}$ $\rightarrow X^{\prime}$ is a morphism, which is the desired one. Then taking a resolution, $g_{1}: \widetilde{X}_{1} \rightarrow X_{1}$, which is a projective morphism if necessary, we may assume from the beginning that $X_{1}$ is nonsingular. On the other hand, by Lemma 4. $5 X_{1}$ is Kähler. This proves the necessity. The sufficiency is obvious. 2) We may assume that $X$ is a subspace of $Y$. By 1) there exists a morphism $h: Y_{1} \rightarrow Y$ with $Y_{1}$ a compact Kähler manifold. Then $X$ is a holomorphic image of a Kähler space $h^{-1}(X)$. 3) Let $h: Y_{1} \rightarrow Y$ be as in 2). Then $X$ is a meromorphic image of $Y_{1}$ and hence $X \in \mathscr{C}$. 4) Let $h: Y_{1} \rightarrow Y$ be as in 2) and $f_{Y}: X \times_{Y} Y_{1} \rightarrow Y_{1}$ the induced map. Then $f_{Y_{1}}$ is a Kähler morphism since $f$ is one. Then by Lemma 4.52) $X \times_{Y} Y_{1}$ is a Kähler space. Hence $X$ is a holomorphic image of a Kähler space $X \times_{Y} Y_{1}$ via the natural projection $X \times{ }_{Y} Y_{1} \rightarrow X$. Q.D.

Remark 4. 4. The author understands that Hironaka once posed the following problem: Let $f: X \rightarrow Y$ be a proper surjective and flat morphism of reduced complex spaces. Then is $Y$ Kähler, if $X$ is Kähler? The affirmative answer to this problem together with the flattening theorem of Hironaka would imply that the class $\mathscr{C}$ is in fact nothing but that 
of compact complex spaces bimeromorphic to compact Kähler manifolds.

Lemma 4. 8. Let $f: Y \rightarrow X$ be a proper surjective morphism of reduced and irreducible complex spaces and $U=\{x \in X ; f$ is flat at every point of $\left.Y_{x}\right\}$. Let $\phi$ be a Hermitian form on $X$ and $q=\operatorname{dim} X$ $-\operatorname{dim} Y$. Then the function, $v(u)=\int_{\left[Y_{u}\right]} \phi^{q}$, which is continuous on $U$ by Corollary 3. 2, is locally bounded on $X$ from above and below by positive constants.

Proof. Take a flattening $\tilde{f}: \widetilde{Y} \rightarrow \widetilde{X}$ of $f$ with flattening morphism $g: \widetilde{X} \rightarrow X$. Let $\widetilde{\phi}$ be the pullback of $\phi$ on $Y$ by the natural map $\pi: \widetilde{Y} \rightarrow Y$. Then by Corollary 3.2 the function, $\widetilde{v}(x)=\int_{\left[\widetilde{Y}_{x}\right]} \widetilde{\phi}^{q}$, is continuous on the whole space $\widetilde{X}$. Hence for any compact subset $K$ of $X, \widetilde{v}$ has the maximum $M$ and the minimum $m \geqq 0$ on $g^{-1}(K)$. On the other hand, since $g$ gives an isomorphism of $g^{-1}(U)$ and $U$, it follows that $\widetilde{v}(x)$ $=v(g(x))$ for $x \in g^{-1}(U)$. Hence $m \leqq v \leqq M$ on $K \cap U$. It remains to show that $m>0$. In fact by the definition of flattening the map $\pi$ restricted to each fiber of $f$ is an embedding into $X$ and hence $\widetilde{v}$ must be positive at every point of $\widetilde{X}$ i.e. $m>0$.

Q.E.D.

Proposition 4. 8. Let $S$ be a complex space and $X$ (resp. $Y$ ) a complex space proper over $S$. Suppose that $Y$ has the property BP over $S$ (cf. 3.4). Then in either of the following two cases $X$ also has the property $B P$ over $S$.

1) $X$ is a subspace of $Y$.

2) There exists a proper surjective $S$-morphism $h: Y \rightarrow X$.

Proof. Let $B$ be any irreducible component of $B_{q}(X / S), q \geqq 0$, and $\pi: B \rightarrow S$ (resp. $\alpha: X \rightarrow S, \beta: Y \rightarrow S$ ) the natural projection. Let $\left\{A_{b} ; b \in B\right\}$ be the associated analytic family of compact $q$-cycles of $X$ over $S$. Let $K$ be any compact subset of $S$. Then we have to show that $\pi^{-}(K)$ is compact. By virtue of Lemma 4.2 as in the proof of Theorem 4.3 we may assume that $q>0$. Let $W=\left\{(b, x) \in B \times X ; x \in\left|A_{b}\right|\right\}$ and $f: W \rightarrow B$ the natural projection. By Lemma 3.1 W is an analytic subset of $B \times X$. Let $\Omega$ be any Hermitian form on $\alpha^{-1}(K)$. Then we shall show: *) The 
function $\lambda(b)=\int_{A_{b}} \Omega^{q}$ is bounded on $\pi^{-1}(K) \cap U$ for some dense Zariski open subset $U$ of $B$. By the continuity of $\lambda$ on $B$ (Cor. 3.3) this would imply that $\lambda(b)$ also is bounded on $\overline{\pi^{-1}(K) \cap U}=\pi^{-1}(K) \cap \bar{U}$ $=\pi^{-1}(K)$. Then since $\left|A_{b}\right|$ are all contained in the compact subset $\alpha^{-1}(K)$ for all $b \in \pi^{-1}(K)$, by Proposition $2.10 \pi^{-1}(K)$ is compact. Hence it remains to show $*$ ) above.

In the case 1$)$ we have the natural $S$-inclusion $B(X / S) \subseteq B(Y / S)$ induced by the inclusion $X \subseteq Y$. Then $B$ is contained in some irreducible component $B^{\prime}$ of $B(Y / S)$. By assumption $B^{\prime}$ is proper over $S$. Let $\pi^{\prime}: B^{\prime} \rightarrow S$ be the natural projection. Take a Hermitian form $\widetilde{\Omega}$ on $\beta^{-1}(K)$ which induces $\Omega$ on $\alpha^{-1}(K)$. Then $\tilde{\lambda}\left(b^{\prime}\right)=\int_{A_{b^{\prime}}} \widetilde{\Omega}$ is bounded on $\pi^{\prime-1}(K)$ and hence $\lambda$ is bounded on $\pi^{-1}(K)$. This shows $*$ ) for $U=B$.

Next we show 2). First we show how to reduce the general case to the case where $W$ is irreducible. Let $W_{j}, 1 \leqq j \leqq s$, be the irreducible components of $W$ and $f_{j}=\left.f\right|_{W_{j}}: W_{j} \rightarrow B$. Then by Lemma 3.11 ) there exists a Zariski open subset $V$ of $B$ and a positive integer $n_{j}$ for each $j$ such that $A_{b}=\sum_{j} n_{j}\left[W_{j b}\right]$. Let $V_{1}$ be the set of nonsingular points of $B$. Then by Lemma 3.12 ) there exist a Zariski open subset $V_{2}$ of $V_{1}$ and for every $j$ an analytic family $\left\{A_{b}^{j} ; b \in B\right\}$ such that $A_{b}^{j}=n_{j}\left[W_{j b}\right]$ for all $b \in V_{2}$. It is clear that these families are over $S$ in the sense of 3.2 , since the original family is. Then by the universality of $B_{q}(X / S)$ we have the unique $S$-morphism $\tau_{j}: V_{2} \rightarrow B_{q}(X / S)$ such that $A_{v}^{j}=A_{\tau_{j}(v)}$, $v \in V_{2}$. Let $B_{j}$ be any irreducible component of $B_{q}(X / S)$ which contains $\tau_{j}\left(V_{2}\right)$. Let $W_{j}^{\prime}$ be the analytic subset of $B_{j} \times X$ associated with the family parametrized by $B_{j}$. By the definition of $B_{j}$ we see readily that $W_{j}^{\prime}$ are irreducible. Assume that $\pi_{j}^{-1}(K)$ is compact with $\pi_{j}: B_{j} \rightarrow S$ the natural projection. Then the functions $\lambda_{j}^{\prime}(b)=\int_{A_{b}} \Omega^{q}$ are bounded on $\pi_{j}^{-1}(K)$ and hence $\lambda_{j}(v)=\int_{A_{v}^{j}} \Omega^{q}$ are bounded on $\pi^{-1}(K) \cap V_{2}$. Therefore $\lambda(v)=\int_{A_{v}} \Omega^{q}=\sum_{j} \int_{A_{v} j} \Omega^{q}=\sum_{j} \lambda_{j}(v)$ is bounded on $\pi^{-1}(K) \cap\left(V \cap V_{2}\right)$ as desired. Hence we may assume that $W$ is irreducible.

First note that $B(X / S)=B\left(X_{\text {red }} / S\right)$ by the definition of $B\left(^{*}\right)$. Hence replacing $X$ by $X_{\text {red }}$ we may assume that $X$ is reduced. Let $\phi$ (resp. $\Omega$ ) be a Hermitian form on $Y$ (resp. X) and $\omega=\phi+h^{*} \Omega$. Then 
(1) is a Hermitian form on $Y$. Then we have to show that $\lambda(b)=\int_{A^{q}} \Omega^{a}$ is bounded on some dense Zariski open subset of $\pi^{-1}(K) \cap B$. First, for every integer $p \geqq 0$ let $X_{p}=\left\{x \in X ; \operatorname{dim} Y_{x} \geqq p\right\}$ and take the minimal $q^{\prime}$ with $\left|A_{b}\right| \nsubseteq X_{q^{\prime}+1}$ for some $b \in B$. Then since $W$ and $B$ are irreducible, there exists an irreducible component, say $X_{1}$, of $X_{q}$, which contains all of $W_{b}, b \in B$. Then replacing $X$ by $X_{i}$ and $Y$ by a suitable irreducible component of $h^{-1}\left(X_{i}\right)$ we may assume that $X$ and $Y$ are irreducible and $q^{\prime}=\operatorname{dim} Y-\operatorname{dim} X$ (cf. the arguments in the proof of 1)). Next take a Zariski open subset $V$ and a positive integer $n$ such that $A_{b}=n\left[W_{b}\right]$ for all $b \in V$. Let $W^{\prime}=\left(h \times i d_{B}\right)^{-1}(W) \subseteq Y \times B$ and $f^{\prime}: W^{\prime} \rightarrow B$ the natural projection. Let $U_{1}=\left\{b \in B ; f^{\prime}\right.$ is flat at every point of $W_{b}^{\prime}$ and $W_{b}^{\prime}$ is reduced $\}$ and $U_{2}$ the set of nonsingular points of $B$. Set $U=U_{1} \cap U_{2} \cap V$. This is a nonempty Zariski open subset of $B$. Then since $f_{U}^{\prime}=f_{W_{U^{\prime}}}^{\prime}$ is flat, every fiber $W_{u}^{\prime}$ of $f_{U}^{\prime}, u \in U$, has pure dimension $q^{\prime}$. Then by Lemma $3.12)$ there is an analytic family $\left\{A_{b}^{\prime} ; b \in U\right\}$ and a Zariski open subset $U_{0}$ of $U$ such that $A_{b}^{\prime}=n\left[W_{b}^{\prime}\right]$ for every $b \in U_{0}$.

We shall see that the family $\left\{A_{b}^{\prime}\right\}$ is bounded on $\pi^{\prime-1}(K) \cap U$ with $\pi^{\prime}: B(Y / S) \rightarrow S$ the structure morphism. Namely we show that there exists a constant $c>0$ such that $\mu(b)=\int_{A_{b^{\prime}}} \omega^{q^{\prime}} \leqq c$ for all $u \in \pi^{\prime-1}(K) \cap U$. Since $\alpha\left(\left|A_{b}\right|\right)=\pi(b)$ for all $b \in B$ and $h$ is an $S$-morphism, it follows that for $u \in U, \beta\left(\left|A_{u}^{\prime}\right|\right)=\beta\left(W_{u}^{\prime}\right)=\beta \bar{h}\left(W_{u}\right)=\beta h\left(\left|A_{u}\right|\right)=\alpha\left(\left|A_{u}\right|\right)=\pi(u)$, where $\bar{h}=h \times i d_{B}$. Thus $\left\{A_{u}^{\prime} ; u \in U\right\}$ is in fact an analytic family of compact $q^{\prime}$-cycles of $Y / S$ in the sense of 3.2. Then by the universality of $B(Y / S)$ we have a unique $S$-morphism $\tau: U \rightarrow B(Y / S)$ such that $A_{u}^{\prime}$ $=A_{\tau(u)}$ for all $u \in U$, where $\left\{A_{b^{\prime}} ; b^{\prime} \in B(Y / S)\right\}$ is the universal family for $Y / S$. Let $B^{\prime}$ be any irreducible component of $B(Y / S)$ which contains $\tau(U)$. Then since $B^{\prime}$ is proper by our assumption, the continuous function $\mu^{\prime}\left(b^{\prime}\right)=\int_{{A_{u}}^{\prime}} \phi^{q^{\prime}}$ is bounded on $\pi^{\prime-1}(K) \cap B^{\prime}$. Hence the function $\mu(u)=\mu^{\prime}(\tau(u))$ also is bounded on $\pi^{-1}(K) \cap U$ as desired.

On the other hand, by Lemma 1.3 we have the inequality $\mu(b)$ $\geqq \int_{A_{b}} v_{b}(x) \Omega^{q}$, where $v_{b}(x)=\int_{\left[W_{x^{\prime}}\right]} \phi^{q^{\prime}}$, defined on some Zariski open subset $U_{b}$ of $\left|A_{b}\right|$. Then since $\left[W_{x}^{\prime}\right]=\left[Y_{x}\right]$, it follows that $v_{b}(x)$ $=\int_{\left[Y_{x}\right]} \phi^{q^{\prime}}$, independent of $b$. The right side of this equality is nothing but the restriction to $U_{b}$ of the function $v$ defined in Lemma 4.7. Hence 
there exists a constant $c^{\prime}>0$ independent of $b$ such that $v_{b}(x)>c^{\prime}$ on $\pi^{-1}(K) \cap U_{b}$. Then it follows that $c \geqq \mu(b)>c^{\prime} \int_{A_{b}} \Omega^{q}=c^{\prime} \lambda(b), \quad b \in U$. Hence $\lambda(b) \geqq c / c^{\prime}$ on $U \cap \pi^{-1}(K)$.

Q.E.D.

Remark 4.5. We need 2) only in the case where $f$ is Kähler. In that case the proof is essentially simpler since the function $v_{b}(x)$ defined in the proof is then constant. In particular Lemma 4.7 is unnecessary though it seems to be of independent interest.

Theorem 4. 9. Let $X$ be a compact reduced complex space which is a meromorphic image of a compact Kähler space. Then X has the property $B P$ and $\bar{D} P$ i.e. every irreducible component of $B(X)$ (resp. $\left.\bar{D}_{X}\right)$ is compact.

Proof. Let $h: Y \rightarrow X$ be a surjective morphism with $Y$ a compact Kähler manifold (c.f. Lemma 4.6 1)). By Theorem $4.5 Y$ has the property $B P$. Then by Proposition 4.82$) X$ also has the property $B P$. The property $\bar{D} P$ then follows from Proposition 3.4. Q.E.D.

\section{§5。 Final Reductions}

5. 1. Let $S$ be a complex space and $X$ a complex space proper over $S$. Let $\mathcal{E}$ be a coherent analytic sheaf on $X$. Then the relative Douady space, $D=D_{X / S}(\mathcal{E})$, associated with $(X / S, \mathcal{E})$ is the complex space over $S$ characterized by the following universal property; there exists a quotient analytic sheaf, $R=\mathcal{E}_{X / S}(\mathscr{R})$, of $\mathcal{E}_{D}$ on $X_{D}$ with the quotient homomorphism, $u=u_{X / S}: \mathcal{E}_{D} \rightarrow \mathscr{R}$ such that it is flat over $D_{X / S}(\mathcal{E})$ and that for every complex space $T$ over $S$ and every quotient homomorphism $u^{\prime}$ : $\mathcal{E}_{T} \rightarrow \mathscr{L}$ for some coherent analytic sheaf $\mathscr{F}$ on $X_{T}$ flat over $T$, there exists a unique $S$-morphism $\tau: T \rightarrow D_{X, S}(\mathcal{E})$ with $\tau^{*} u=u^{\prime}$. Then we call $u$, or $\mathscr{R}$ itself, the universal quotient of $\mathcal{E}$. The existence of such $D_{X / S}(\mathcal{E})$ and hence of $R_{X / S}(\mathcal{E})$ was shown by Douady in [5] when $S$ is a point, the generalization to the relative case being due to Pourcin [17]. If $\mathcal{E}=\mathcal{O}_{X}$, then as usual we identify $D_{X / S}\left(\Theta_{X}\right)$ with $D_{X / S}$ so that $Z=Z_{X / S}$ is the subspace of $X_{D_{X / S}\left(\mathcal{O}_{X}\right)}$ corresponding to $\mathscr{R}_{X / S}\left(\mathcal{O}_{X}\right)$ (cf. 3.3). 
Notation. If $D_{\alpha}$ is an irreducible component of $D_{X / S}(\mathcal{E})$, then we often write $X_{a}, Z_{\alpha}, \mathcal{E}_{a}, \mathcal{R}_{\alpha}$ etc. instead of $X_{D_{a}}, Z_{D_{\alpha}}, \mathcal{E}_{D_{\alpha}}, \mathcal{R}_{D_{\alpha}}$ etc.

Let $X$ be a complex space and $B$ a locally closed analytic subspace of $X$. Then $B$ is called Zariski locally closed if $B$ is a Zariski open subset of some analytic subspace $B_{0}$ of $X$. In that case there exists the minimal such $B_{0}$, which we call the analytic closure of $B$ in $X$. Next, let $X$ and $Y$ be complex spaces. Then a meromorphic map from $X$ to $Y$ is an equivalence class of morphisms $f: U \rightarrow Y$ defined on some dense Zariski open subset $U=U(f)$ of $X$ such that the graph $\Gamma_{f}$ of $f$ in $U \times Y$ is Zariski locally closed in $X \times Y$. Here two such $f_{i}: U_{i} \rightarrow Y, i=1,2$, is called equivalent if $f_{1}=f_{2}$ on $U_{1} \cap U_{2}$. We denote a meromorphic map defined by a morphism $f: U \rightarrow Y$ as above by $\bar{f}: X \rightarrow Y$, and call the analytic closure of $\Gamma_{f}$ in $X \times Y$ the graph of $\bar{f}$. If both $X$ and $Y$ are complex spaces over $S$, then a meromorphic map $\bar{f}: X \rightarrow Y$ as above is said to be over $S$, or an $S$-meromorphic map, if $f$ is an $S$-morphism. Suppose further that there exists an $S$-morphism $\alpha: Y \rightarrow X$. Then an $S$-meromorphic map $\bar{f}$ : $X \rightarrow Y$ is called a meromorphic $S$-section if $\alpha f=i d_{t}$. We call an $S$-meromorphic map $\bar{f}: X \rightarrow Y$ weakly defined if there exists a proper $S$-bimeromorphic morphism $h: \widetilde{X} \rightarrow X$ such that $f h$ is a morphism, or more precisely the meromorphic map $\overline{f h}: X \rightarrow Y$ defined by $f h$ on $f^{-1}(U)$ is represented by such. It is easy to see that in this case if $f$ is generically surjective and $X$ is proper over $S$, then $Y$ also is proper over $S$. Finally we note that the above definitions are all independent of the particular representative $f$ and depends only on the meromorphic map $\bar{f}$ which $f$ defines. In particular it makes sense to speak of a graph of a meromorphic map.

Lemma 5. 1. Let $S$ be a complex space, $X$ a complex space proper over $S$, and $\mathcal{E}$ a coherent analytic sheaf on $X$. Let $T$ be a reduced complex space over $S$. Let $u: \mathcal{E}_{T} \rightarrow \mathscr{F}$ be a quotient of $\mathcal{E}_{T}$ on $X_{T}$ and $U$ any Zariski open subset of $T$ such that $\mathscr{F}$ is flat over $U$. Let $h: U \rightarrow D_{X / S}(\mathcal{E})$ be the morphism induced by the universality of $D_{X / S}(\mathcal{E})$. Then $h$ defines a reakly defined $S$-meromorphic map $\bar{h}$ : $T \rightarrow D_{X / S}(\mathcal{E})$.

Proof. Take a flattening, $f_{\tilde{T}}: X_{\widetilde{T}} \rightarrow \widetilde{T}$, of $\mathscr{F}$ induced by a proper 
bimeromorphic morphism $\phi: \widetilde{T} \rightarrow T$. Let $\widetilde{\mathscr{F}}$ be the $\mathscr{F}_{\widetilde{T}}$ modulo $f_{\widetilde{T} \text {-torsion }}$ $[14,4.2 .1]$. $\widetilde{\mathscr{I}}$ is a quotient of $\varepsilon_{T}$ on $X_{T}$ and is flat over $T$. Hence by the universality of $D_{X / S}(\varepsilon)$ we have a unique $S$-morphism $\tau$ : $T \rightarrow D_{X / S}(\mathcal{E})$ such that $\widetilde{\mathscr{I}} \cong \mathcal{R}_{\tilde{T}}$, where $\widetilde{T}$ is over $S$ by $\beta \cdot \phi$ with $\beta: T \rightarrow S$. Moreover $\tau=h \cdot \phi$ on $\phi^{-1}(U)$. This shows that $h$ is $S$-meromorphic and is weakly defined.

Q.E.D.

The purpose of this section is to prove the following theorems.

Theorem 5. 2. Let $f: X \rightarrow S$ be a proper Kähler morphism and $\mathcal{C}$ a coherent analytic sheaf on $X$. Then every irreducible component of $D_{X / S}(\mathcal{E})_{\text {red }}$ is proper over $S$. In particular if $X$ is a compact Kähler space, then every irreducible component of the Douady space $D_{X \text {.red }}$ is compact.

Theorem 5. 3. Let $X$ be a compact complex space such that $X_{\text {red }}$ is a meromorphic image of a compact Kähler space. Let $\varepsilon$ be a coherent analytic sheaf on $X$. Then every irreducible component of $D_{X}(\mathcal{E})$ is compact.

Our proof of Theorems 5.2 and 5.3 consists in reducing the problem in three steps (Lemmas 5.8,5.9 and 5.7) to the case of $\bar{D}_{X / S}$ for which we have already proved Proposition 3. 4.

5. 2. The purpose of this paragraph is to formulate and prove Lemma 5.7 below. Let $X$ be a complex space and $\mathcal{E}$ a coherent analytic sheaf on $X$. For every integer $r \geqq 0$ let Grass ${ }_{r}\left(\mathcal{E}^{\prime}\right)$ be the Grassmann variety of locally free quotients of rank $r$ of $\mathcal{E}$ (see [11, V.2] for the definition). Let $\alpha: \operatorname{Grass}_{r}(\mathcal{E}) \rightarrow X$ be the natural projection and $u_{0}: \alpha^{*} \mathcal{E} \rightarrow \mathscr{F}_{0}$ the universal locally free quotient. Suppose now that $X$ is reduced. Let $u$ : $\mathcal{E} \rightarrow \mathscr{F}$ be any quotient of $\mathcal{E}$ on $X$, i.e. a quotient analytic sheaf $\mathscr{F}$ of $\mathcal{E}$ with the quotient map $u$. Then there exists a dense Zariski open subset $U$ of $X$ such that $\mathscr{F}$ is locally free of finite rank, say $r_{i}$, on each connected component $U_{i}$ of $U$. Assume that $r=r_{i}$ for some $r$ and all $i$. Then by the universality of $u_{0}$ we have the unique section $s_{U}: U \rightarrow \operatorname{Grass}_{r}(\mathcal{E})_{U}$ such that $s_{U}^{*} u_{0}=u$. Moreover it is well-known and easy to verify that 
$s_{U}$ extends to a unique meromorphic section $s: X \rightarrow \operatorname{Grass}_{r}(\mathcal{E})$ (cf. [18, Prop. 3. 4]). We call this $s$ the meromorphic section associated to $u$ and denote it by $s=s(u)$ or by $s=s(\mathscr{F})$ with $u$ understood.

Lemma 5. 4. Let $X$ and $\mathcal{E}$ be as above. Then the map $u \rightarrow s(u)$ defined above gives a bijective correspondence between the set of torsion free $e^{4)}$ quotients $u: \mathcal{E} \rightarrow \mathscr{F}$ and that of meromorphic sections to $\alpha$.

Proof. First we show that $u: \mathcal{E} \rightarrow \mathscr{F}$ is uniquely determined by $s(u)$, if $\mathscr{F}$ is torsion free. So let $u_{i}: \mathcal{E} \rightarrow \mathscr{F}_{i}, i=1,2$, be the torsion free quotients of $\mathcal{E}$ with $s=s\left(u_{1}\right)=s\left(u_{2}\right)$. Let $\mathscr{F}_{i}^{\prime}$ be the kernels of $u_{i}$. Assume first that $\mathscr{F}_{1}^{\prime} \subseteq \mathscr{F}_{2}^{\prime}$. Then we have the natural surjection $u_{12}: \mathscr{F}_{1} \rightarrow \mathscr{F}_{2}$ with $u_{12} u_{1}=u_{2}$. Let $U$ be the Zariski open subset of $X$ on which $s$ is defined. Then by our assumption $u_{1}=u_{2}$ on $U$ so that the kernel of $u_{12}$ has support in $X-U$. Since $\mathscr{F}_{1}$ is torsion free, it must then vanish identically. Hence $u_{1}=u_{2}$ on the whole $X$. In the general case let $\mathscr{F}^{\prime}=\mathscr{F}_{1}^{\prime}+\mathscr{F}_{2}^{\prime}$ and $\mathscr{F}=\mathcal{E} / \mathscr{F}^{\prime}$. Then clearly $s=s(\mathscr{F})$. Further by the above arguments it follows that $\mathscr{F}_{1}=\mathscr{I}_{1}=\mathscr{F}_{2}$ and hence $u_{1}=u_{2}$.

Next take any meromorphic section $s: X \rightarrow \operatorname{Grass}_{r}(\mathcal{E})$. We show that there exists a torsion free quotient $u: \mathcal{E} \rightarrow \mathscr{F}$ with $s=s(u)$. Let $\widetilde{X}$ be the graph of $s$ in $X \times \operatorname{Grass}_{r}(\mathcal{E})$ and $\widetilde{s}: \widetilde{X} \rightarrow \operatorname{Grass}_{r}(\mathcal{E})$ (resp. $\sigma: \widetilde{X} \rightarrow X$ ) the natural projection. Let $\widetilde{u}_{0}: \tilde{s}^{*} \alpha^{*} \mathcal{E} \rightarrow \tilde{s}^{*} \mathscr{F}_{0}$ be the pull-back of the universal quotient $u_{0}$ by $\tilde{s}$. Taking the direct image by $\sigma$ and noting that $\sigma=\alpha \tilde{s}$, $\widetilde{u}_{0}$ defines a homomorphism $\sigma_{*} \widetilde{u}_{0}: \sigma_{*} \sigma^{*} \mathcal{E} \rightarrow \sigma_{*} \tilde{s}^{*} \mathscr{F}_{0}$. Let $r: \mathcal{E} \rightarrow \sigma_{*} \sigma^{*} \mathcal{E}$ be the natural homomorphism. Define $\mathscr{F}=\left(\sigma_{*} \widetilde{u}_{0}\right) \gamma(\mathcal{E})$ and $u: \mathcal{E} \rightarrow \mathscr{F}$ to be the natural map. Then we see easily that $s=s(\mathscr{F})$. Finally replacing $\mathscr{F}$ by $\mathscr{F} /$ Tor $\mathscr{F}$ with Tor $\mathscr{F}$ the torsion part of $\mathscr{F}$ if necessary, without affecting the condition $s=s(\mathscr{F})$, we may assume that $\mathscr{F}$ is torsion free.

Q.E.D.

Lemma 5. 5. 1) Let $f: X \rightarrow T$ and $f^{\prime}: X^{\prime} \rightarrow T$ be proper morphisms of complex spaces. Suppose that every fiber of $f$ has pure dimension $q \geqq 0$ and $T$ is reduced. Let $Z$ be a subspace of $X \times{ }_{T} X^{\prime}$. Define the

4) $\mathscr{I}$ is called torsion free if for every $x \in X$ and every non zero divisor $a \in \mathcal{O}_{x, x}$, the homothety $\mathscr{F}_{x} \rightarrow \mathscr{F}_{x}$ induced by $a$ is injective (cf. EGA I 7.4.7). 
subset $M_{0}$, of $T$ by $M_{0}=\left\{t \in T ; Z_{t}\right.$ is the graph of a meromorphic map from $X_{t}$ to $\left.X_{t}^{\prime}\right\}$. Then $M_{0}$ is a Zariski open subset of $T$.

2) In 1) suppose further that $f$ is flat and there exists an $S$-morphism $g: X^{\prime} \rightarrow X$ such that $f g=f^{\prime}$. Then the set $M$ defined by $m=\{t \in T$; $Z_{t}$ is the graph of a meromorphic section to $\left.g_{t}=\left.g\right|_{x_{t}^{\prime}}\right\}$ is Zariski locally closed in $T$.

Proof. 1) Let $\pi: Z \rightarrow X$ be the natural projection and $\iota: \mathcal{O}_{X} \rightarrow \pi_{*} \mathcal{O}_{Z}$ the natural morphism. Define $A_{1}=\left\{x \in X ; \operatorname{dim} \pi^{-1}(x) \geqq 1\right\}$ and $A_{2}=$ $\operatorname{supp}\left(\pi_{*} \mathcal{O}_{z} / \iota \mathcal{O}_{X}\right)$. Set $A=A_{1} \cup A_{2}$. Let $N_{0}=\left\{t \in T ; \operatorname{dim} A_{t} \geqq \mathrm{q}\right\}$. By Remmert $N_{0}$ is an analytic subset of $T$. We show that $U=T-N_{0}$ coincides with $M_{0}$. We first note that $\left(\pi_{*} \mathcal{O}_{Z} / \iota \Theta_{X}\right) \otimes \mathcal{O}_{X_{t}} \cong \pi_{*} \Theta_{Z} \otimes\left(\Theta_{X_{t}} / c_{t} \Theta_{X_{t}}\right)$ $\cong \pi_{t_{1}} \mathcal{O}_{z_{t}} / \iota_{t} \mathcal{O}_{X_{t}}$, where $\pi_{t}=\pi_{Z_{t}}: Z_{t} \rightarrow X_{t}$ and $\iota_{t}: \mathcal{O}_{X_{t}} \rightarrow \pi_{t_{m}} \mathcal{O}_{Z_{t}}$ is the natural map. hence by Nakayama $x \notin A_{2}$ if and only if $\pi_{*} \mathcal{O}_{z_{t}}=\mathcal{O}_{x_{t}}$. This implies that $x \notin A$ if and only if $\pi_{l}^{-1}$ is defined at $x$ and $\pi_{t}$ is isomorphic at $z=\pi_{t}^{-1}(x)$. Then since every fiber of $f$ has pure dimension $q$, we see readily that $U$ coincides with the set $\left\{t \in T ; \pi_{t}: Z_{t} \rightarrow X_{t}\right.$ is bimeromorphic $\}$ and hence with $M_{0}$.

2) Let $I$ be the graph of $g$ in $X^{\prime} \times{ }_{T} X$. Put $Y=p r_{13}\{(X \times \Gamma) \cap(Z$ $\times X)\}$, where $p r_{13}: X \times{ }_{T} X^{\prime} \times{ }_{T} X \rightarrow X \times{ }_{T} X$ is the natural projection. Since $p r_{13}$ is proper, $Y$ is an analytic subspace of $X \times{ }_{T} X$. Let $\Delta \subseteq X \times{ }_{T} X$ be the diagonal and define $N=\left\{t \in T ; Y_{t} \subseteq J_{t}\right\}$. Then since the natural map $\Delta \rightarrow S$ is flat, it follows immediately from [17, Prop. 1] (putting $\mathcal{E}=\mathcal{O}_{\Delta}$ and $\mathscr{F}=\mathcal{O}_{Y \cap \Delta}$ there) that $N$ is an analytic subset of $T$. Finally put $V=M_{0} \cap N$. Then by 1 ) it suffices to show that $V=M$. In fact one sees easily that both coincides with the set $\left\{t \in M_{0} ; g s_{t}=\right.$ identity on some dense Zariski open set of $X_{t}$ on which $s_{t}$ is defined\}, where $s_{t}$ is the meromorphic map corresponding to $t \in M_{0}$.

Q.E.D.

Lemma 1.4 and a modification of its proof yield the following lemma.

Lemma 5. 6. Let $f: X \rightarrow S$ be a proper and surjective morphism of reduced complex spaces and $\mathscr{R}$ a coherent analytic sheaf on $X$ which is fat over $S$ by $f$. Suppose that $X$ is pure dimensional, and $S$ is irreducible. Then the following conditions are equivalent. 
1) There exists a point $s \in S$ such that $R_{s}$ is torsion free as an $\mathcal{O}_{X_{s}}$ module.

2) The set $U=\left\{s \in S ; \mathscr{R}_{s}\right.$ is torsion free $\}$ is dense and Zariski open in $S$.

3) $\mathscr{R}$ is torsion free on $X$.

Proof. We shall show that the set $U$ in 2) is Zariski open in $S$, which would establish the equivalence of 1) and 2). First we recall a general fact. Let $\mathscr{F}$ be a coherent analytic sheaf on a complex space $Y$ on pure dimension $n$. Let $S_{k}(\mathscr{F})=\left\{y \in Y\right.$; $\left.\operatorname{depth}_{y} \mathscr{F} \leqq k\right\}, k \geqq 0$, which is an analytic subset of $Y$. Suppose that supp $\mathscr{F}$ coincides with $Y_{\text {red. }}$ Then the following conditions are equivalent (cf. EGA IV 5.7);1) $\mathscr{F}$ is torsion free, for every $y \in Y 2) \mathscr{F}_{y}$ has no embedded primes 3) $\operatorname{dim}_{y} S_{k}\left(\mathscr{F}^{\prime}\right)$ $<k$ for all $k \leqq n-1$. Now returning to our situation let $S_{k}(\mathscr{R}, f)=\{x$ $\left.\in X ; \operatorname{depth}_{x} \mathscr{R}_{f(x)} \leqq k\right\}$ and $B_{k}(\mathscr{R}, f)=\left\{x ; \operatorname{dim}_{x} S_{k}(\mathscr{R}, f) \cap X_{f(x)} \geqq k\right\}$. From the flatness of $\mathscr{R}$ it follows immediately that either of the three conditions implies that $\operatorname{supp} \mathscr{R}=X$ so that $\operatorname{supp} \mathcal{R}_{s}=X_{s, \text { red }}$ for all $s \in S$. Hence by the equivalence of 1 ) and 3 ) above our assertion follows from the analyticity of $B_{k}(\mathscr{R}, f)$, which in turn is a consequence of that of $S_{k}(R, f)$, shown by Banica in [2], and the fact that the set $\left\{x ; \operatorname{dim}_{x} T\right.$ $\left.\cap X_{f(x)} \geqq k\right\}$ is analytic for every analytic subset $T$ of $X$ as was noted in [2]. The equivalence of 2) and 3) can be proved almost analogously to that of 2) and 3) of Lemma 1.4. In fact, let $n=\operatorname{dim} X, m=\operatorname{dim} S$ and $q=n-m$. Suppose that $q>0$, leaving the case $q=0$ to the reader. Now for any smooth point $x \in X$ with $f(x)$ a smooth point of $S$ we have $\operatorname{depth}_{x} \mathscr{R}=\operatorname{depth}_{x} \mathscr{R}_{f(x)}+m$, so that $S_{k}(\mathscr{R}, f)=S_{k+m}(\mathscr{R})$ for every $k \geqq 0$ at $x$. Let $B_{k}(R)=\left\{x ; \operatorname{dim}_{x} S_{k}(\mathscr{R}) \geqq k\right\}$. Then as in the proof of Lemma 1. 4, for $x \in X$ as above $A(\mathscr{R})=\bigcup_{n-1 \geq k \geq m} B_{k}(\mathscr{R})$ vanishes at $x$ if and only if $A_{f}(R)=\bigcup_{0 \leqq k \leqq q-1} B_{k}(R, f)$ does. Then by the above mentioned equivalence it follows that 3) implies 2) and 2) implies that $R$ is torsion free on $f^{-1}(U)$ for a Zariski open subset $U$ of smooth points of $S$. Then the rest of the proof is done as in the last part of the proof of Lemma 1.4.

Q.E.D.

Lemma 5. 7. Let $X$ and $S$ be reduced and irreducible complex 
spaces and $f: X \rightarrow S$ a proper and flat morphism. Let $\mathcal{E}$ be a coherent analytic sheaf on $X$ and $Y=X \times{ }_{s} \operatorname{Grass}_{r}(\varepsilon)$ for some $r>0$. Then for every irreducible component, $D_{\alpha}$, of $D_{X / S}(\mathcal{E})_{\text {red }}$ such that $X_{\alpha}$ is reduced and $\mathscr{R}_{\alpha}$ is torsion free of rank $r$ on $X_{\alpha}$ (see 5.1 for the notations) there exist an analytic subset, $E_{\alpha}$, of $\bar{D}_{Y / S}$ and a weakly defined $S$ bimeromorphic map $\tau: E_{\alpha} \rightarrow D_{\alpha}$. Moreover if $X \rightarrow S$ is a Kähler morphism, then $E_{a}$ is proper over $S$.

Proof. Let $\pi: \operatorname{Grass}_{r}(\mathcal{E}) \rightarrow X$ be the natural projection. Let $s=s\left(\mathscr{R}_{\alpha}\right): X_{\alpha} \rightarrow \operatorname{Grass}_{r}\left(\mathcal{E}_{\alpha}\right)=\operatorname{Grass}_{r}(\mathcal{E})_{\alpha}$ be the meromorphic section corresponding to $R_{\alpha}$ by Lemma 5.4. Then the graph $\Gamma$ of $s$ is a subspace of $X_{\alpha} \times_{D_{\alpha}} \operatorname{Grass}_{r}(\mathcal{E})_{\alpha} \cong Y_{\alpha}$. On the other hand, by Frisch [6] there exists a dense Zariski open subset $U$ of $D_{a}$ such that $\Gamma$ is flat over $U$, where $\Gamma$ is naturally over $U$ as a subspace of $Y_{\alpha}$. Then by the universality of $D_{Y / S}$ we have a unique $S$-morphism $h: U \rightarrow D_{Y / S}$ such that $\Gamma_{U}=Z_{U}$, where $Z=Z_{Y / S}$ and $U$ is over $D_{Y / S}$ by $h$. In view of Lemma 5.51 ), restricting $U$ if necessary we may assume that for every point $d \in U, s$ defines a meromorphic map $s_{d}: X_{\alpha d} \rightarrow \operatorname{Grass}_{r}\left(\mathcal{E}_{\alpha}\right)_{d}=\operatorname{Grass}_{r}\left(\mathcal{E}_{\alpha, d}\right)$ such that $\Gamma_{d}$ is its graph. Further since $s$ is a section, $s_{d}$ also is a meromorphic section to $\pi_{d}$ : $\operatorname{Grass}_{r}\left(\mathcal{E}_{\alpha}\right)_{d} \rightarrow X_{\alpha d}$.

Let $M=\left\{\bar{d} \in D_{Y / S} ; Z_{Y / S, \bar{d}}\right.$ corresponds to a graph of a meromorphic section to $\left.\pi_{\bar{a}}: \operatorname{Grass}_{r}(\mathcal{E})_{\bar{d}} \rightarrow X_{\bar{d}}\right\}$. By Lemma 5.5 $M$ is Zariski locally closed in $D_{Y / S}$. On the other hand, by what we have seen above we get that $h(U) \subseteq M$.

Let $\bar{M}$ be the analytic closure of $M$ in $D_{Y / S}$. Then $Z_{\bar{Z}} \subseteq Y_{\bar{M}} \cong X_{\bar{M}}$ $\times_{\bar{M}} \operatorname{Grass}_{r}\left(\mathcal{E}_{\bar{M}}\right)$ is easily seen to be the graph of a unique meromorphic section $s_{\bar{M}}: X_{\bar{M}} \rightarrow \operatorname{Grass}_{r}\left(\hat{E}_{\bar{M}}\right)$. Let $\bar{M}_{1}$ be the minimal analytic subset of $\bar{M}$ containing $h(U)$ and $s_{1}=\left.s_{\bar{M}}\right|_{x_{\bar{M}}}$. One sees readily that $Z_{\bar{M}_{1}}$ is reduced so that $\bar{M}_{1} \subseteq \bar{D}_{Y / S}$.

Let $u: \mathcal{E}_{\bar{M}_{1}} \rightarrow \mathscr{F}$ be the torsion free quotient of $\mathcal{E}_{\bar{M}_{1}}$ corresponding to $s_{1}$ by Lemma 5.4. This is welldefined since $X_{\bar{M}_{1}}$ is reduced as follows from Lemma 1.4. Let $W$ be the Zariski open subset of $\bar{M}_{1}$ such that $\mathscr{F}$ is flat over $W$. Then by the universality of $D_{X / S}(\mathcal{E})$ there is a unique $S$-morphism $\tau: W \rightarrow D_{X / S}(\mathcal{E})$ such that $\mathscr{Z}_{W} \cong \mathscr{R}_{W}$, where $W$ is over $S$ by $\tau$. We show that $h$ gives an $S$-bimeromorphic map of $U$ to $\bar{M}_{1}$ 
and $\tau$ its inverse. We take a nonempty Zariski open subset $U_{1}$ (resp. $W^{\prime}$ ) of $U$ (resp. W) such that $X_{u}=X_{\alpha, u}$ (resp. $X_{w}=X_{W, w}$ ) is reduced and $\mathscr{R}_{u}$ (resp. $\mathscr{F}_{w}$ ) is torsion free for every $u \in U_{1}$ (resp. $w \in W_{1}^{\prime}$ ) (Lemmas 1. 4 and 5.6). Set $W_{1}=W_{1}^{\prime} \cap M$. Then for every $\iota \in U_{1}\left(\right.$ resp. $\left.w \in W_{1}\right)$ the meromorphic section $s_{u}: X_{u} \rightarrow \operatorname{Grass}_{r}\left(\mathcal{E}_{u}\right)$ (resp. $s_{1 w}: X_{w} \rightarrow \operatorname{Grass}_{r}\left(\mathcal{E}_{w}\right)$ ) corresponds to $\mathscr{R}_{u}$ (resp. $\mathscr{E}_{w}$ ) in the sense of Lemma 5.4 on the reduced space $X_{u}$ (resp. $\left.X_{w}\right)$. Put $U_{2}=h^{-1}\left(W_{1}\right) \cap U_{1}$, which is nonempty by our choice of $\bar{M}_{1}$. Then by the above remark combined with the isomorphism $\mathscr{R}_{\tau h(u)} \cong \mathscr{F}_{h(u)}$ we infer readily that $\tau h$ is the identity on $U_{2}$. In particular $\tau(W) \subseteq D_{\alpha}$. Then if we set $W_{2}=\tau^{-1}(U) \cap W_{1}$, by a similar reasoning together with the isomorphism $\Gamma_{\tau(w)}=Z_{h \tau(w)}$ we see that $h \tau$ is the identity on $W_{2}$. This proves our assertion. Finally putting $E_{\alpha}=\bar{M}_{1}$, $\tau$ is weakly defined by Lemma 5.1 .

Next suppose that $X$ is Kähler over $S$. We show that $\bar{M}_{1}$ is proper over $S$. Let $\tilde{\pi}: Y \rightarrow X \times{ }_{S} X$ with $\tilde{\pi}=i d_{X} \times{ }_{s} \pi, \Delta$ the diagonal of $X \times{ }_{S} X$ and $\tilde{\Delta}=\tilde{\pi}^{-1}(\Delta)$. Then as is clear from the proof of Lemma $5.5 Z_{\bar{M}_{1}} \subseteq \tilde{\Delta}_{\bar{M}_{1}}$. In other words, we may consider $\bar{M}_{1}$ naturally as a subspace of $D_{\tilde{\Delta} / \mathrm{s}}$. Since $\tilde{\Delta}$ is projective over $\Delta$ and $\Delta$ is $S$-isomorphic to $X$, by Lemma 4.4 together with Proposition 3. 4 we see that $\bar{M}_{1}$ is proper over $S$. Q.E.D.

5. 3. Let $X$ be a complex space and $\varepsilon$ be a coherent analytic sheaf on $X$. Let $\mathcal{A}(\mathcal{E})$ be the sheaf of annihilators of $\mathcal{E}$ on $X$. This is a coherent sheaf of ideals of $\mathcal{O}_{X}$. Let supp $\mathcal{E}$ be the support of $\mathcal{E}$. Then we define the subspace, $S(\mathcal{E})$, of $X$ by $S(\mathcal{E})=\left(\operatorname{supp} \mathcal{E}, \mathcal{O}_{X} / \mathcal{A}(\mathcal{E})\right)$. Let $S$ be a complex space and suppose that $X$ is over $S$ with the morphism $f: X \rightarrow S$. Then we write $\operatorname{dim} \mathcal{E} / S=\operatorname{dim} \operatorname{supp} \mathcal{E}-\operatorname{dim} S$ and if $\mathcal{E}=\mathcal{O}_{X}$, then $\operatorname{dim} X / S=\operatorname{dim} \mathcal{O}_{X} / S$.

Lemma 5. 8. Let $f: X \rightarrow S$ and $\mathcal{E}$ be as in Theorem 5. 2. Then for every irreducible component $D_{\alpha}$ of $D_{x / S}(\mathcal{E})_{\mathrm{red}}$ with $\operatorname{dim} \mathscr{R}_{\alpha} / D_{\alpha}$ $=q \geqq 0$, there exist 1) an irreducible component, $T$ of $D_{X / S, \text { red }}$ such that $Z_{T}$ is reduced and $\operatorname{dim} Z_{T} / T=q$, where $Z=Z_{X / S}$, 2) a finite number of coherent analytic sheaves, $\mathcal{E}_{k}, 0 \leqq k \leqq n$, on $\left.Z_{T}, 3\right)$ an irreducible component $B_{k}$ of $D_{Z_{T} / T}\left(\mathcal{E}_{k}\right)$ for each $k$ such that $\left(Z_{T}\right)_{B_{k}}$ is reduced, and finally 4) a bimeromorphic $S$-morphism $h: B_{0} \times{ }_{T} \cdots \times{ }_{T} B_{n} \rightarrow D_{\alpha}$. 
Proof. Let $\mathcal{G}$ (resp. $\mathcal{G}$ ) be the ideal sheaf of $S\left(\mathscr{R}_{\alpha}\right)$ (resp. supp $\mathscr{R}_{\alpha}$ ) in $X_{\alpha}$. Define $\mathcal{R}_{k}=\mathscr{I}^{k} \mathscr{R}_{\alpha} / \mathcal{I}^{k+1} \mathscr{R}_{\alpha}, k \geqq 0$, where $\mathscr{I}^{0}=\mathcal{O}=\mathcal{O}_{X_{\alpha}} . \quad$ By definition we have the following exact sequences on $X_{\alpha}$

$$
0 \rightarrow \mathscr{R}_{k} \rightarrow \mathscr{R} / \mathcal{I}^{k+1} \mathscr{R} \rightarrow \mathscr{R} / \mathfrak{I}^{k} \mathscr{R} \rightarrow 0, \quad \mathscr{R}=\mathscr{R}_{\alpha} .
$$

Now take $n>0$ sufficiently large so that $\mathscr{g} \supseteqq \mathscr{I}^{n+1}$ on the general fiber of $S\left(\mathscr{R}_{\alpha}\right) \rightarrow D_{\alpha}$. By Frisch [6] we can find a nonempty Zariski open subset $U$ of $D_{\alpha}$ such that $\mathcal{O} / \mathcal{G}$ and $\mathscr{R}_{k}, 0 \leqq k \leqq n+1$, are all flat over $U$. Moreover restricting $U$ if necessary we may assume that $g \supseteq g^{n+1}$ on $f^{-1}(U)$. Note that by the above exact sequences we see that $\mathscr{R} / \mathcal{I}^{k} \mathscr{R}$ also are flat over $U$ for $0 \leqq k \leqq n+1$. Since $O / \mathcal{G}$ is flat over $U$, by the universality of $D_{X / S}$ we have a unique $S$-morphism $\varphi: U \rightarrow D_{X / S}$ such that $\left(\operatorname{supp} \mathcal{R}_{\alpha}\right)_{U} \cong Z_{U}$. Let $T$ be any irreducible component of $D_{X / S \text {, red }}$ containing $\varphi(U)$. Then since $Z_{U}=\left(\operatorname{supp} R_{\alpha}\right)_{U}$ is reduced and $Z_{T}$ is flat over $T$, $Z_{T}$ is reduced (cf. the proof of Lemma 1.4 noting that the former condition implies that $Z_{T}$ is reduced in a neighborhood of $p^{-1}(\varphi(U))$ with $p: Z_{T} \rightarrow T$ the natural projection).

Now let $\mathcal{I}_{0}$ be the ideal sheaf of $Z_{T}$ in $X_{T}$ and $\mathcal{E}_{k}=\mathcal{G}_{0}^{k} \mathcal{E}_{T} / \mathcal{I}_{0}^{k+1} \mathcal{E}_{T}$. We consider the relative Douady space, $D_{k}=D_{Z_{T / T}}\left(\mathcal{E}_{k}\right)$, associated with the pair $\left(Z_{T} / T, \mathcal{E}_{k}\right)$. Then since $\left(\mathcal{E}_{k}\right)_{U}=\mathcal{I}^{k} \mathcal{E}_{U} / \mathcal{J}^{k+1} \mathcal{E}_{U^{T}}$ and $\mathscr{R}_{k}$ are flat quotients of the latter over $U$, by the universality of $D_{k}$ we have a unique $T$-morphism $\tau_{k}: U \rightarrow D_{k}$, i.e. $\pi_{k} \tau_{k}=\varphi$ with $\pi_{k}: D_{k} \rightarrow T$ the projection, such that $\mathscr{R}_{Z_{T / T}}\left(\mathcal{E}_{k}\right)_{U}=\left(\mathscr{R}_{k}\right)_{U}$. Let $B_{k}$ be any irreducible component of $D_{k \text {,red }}$ containing $\tau_{k}(U)$. Then in the same way as for $Z_{T}$ one sees easily that $\left(Z_{T}\right)_{B_{k}}$ is also reduced. Define $\tau=\tau_{1} \times{ }_{T} \cdots \times_{T} \tau_{n}: U \rightarrow B=B_{1} \times{ }_{T} \cdots \times_{T} B_{n}$ 。 Then by the above exact sequences together with the flatness of $\mathscr{R} / \mathscr{I}^{k} \mathscr{R}$ over $U$ we infer readily that $\tau$ is in fact injective.

Next we construct a family of quotients of $\varepsilon_{k}$ parametrized by $B$. Let $\mathscr{R}_{(k)}=\left(\mathcal{R}_{Z_{T^{\prime} T}}\left(\varepsilon_{k}\right)\right)_{B}$ and $\tilde{\mathcal{I}}$ the ideal sheaf of $Z_{B}$ in $X_{B}$. Then we shall define inductively the following successive quotients, $\mathcal{E}_{B} \rightarrow \mathscr{R}^{(n)}$ $\rightarrow \cdots \rightarrow \mathscr{R}^{(0)}$, of $\mathcal{E}_{B}$ on $X_{B}$ which fit into the following commutative diagram of exact sequences

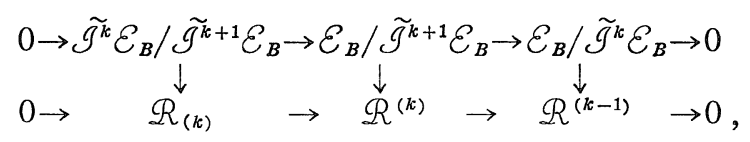


with the vertical arrows surjective. First put $\mathscr{R}^{(0)}=\mathscr{R}_{(0)}$. Next assume that $k>0$ and $\mathscr{R}^{(j)}$ are all defined for $j<k$. Then $\mathscr{R}^{(k)}$ can be defined uniquely by the commutativity of the above diagram. We set $\widetilde{R}=\mathscr{R}^{(n+1)}$. Then $\widetilde{R}$ is naturally the quotient of $\mathcal{E}_{B}$ on $X_{B}$. Moreover by the second line of the above exact sequences together with the flatness of $\mathscr{R}_{(k)}$ we see that $\widetilde{R}$ is flat over $B$. Then by the universality of $D_{x / S}(\mathcal{E})$ we have a unique $S$-morphism $h: B \rightarrow D_{X / S}(\mathcal{E})$ such that $\widetilde{R} \cong \mathscr{R}_{B}, \mathcal{R}=\mathscr{R}_{X / S}(\mathcal{E})$. On the other hand, by our construction we see immediately that $h \tau=i d_{U}$. It follows then that $h(B) \subseteq D_{\alpha}$. Analogously we see that $\tau h$ is the identity on $h^{-1}(U)$. Hence $h$ is bimeromorphic. This proves Lemma 5.8.

Lemma 5.9. Let $f ; X \rightarrow S$ and $\mathcal{E}$ be as in Theorem 5.2. Suppose that $f$ is flat, $X$ is reduced and $\operatorname{dim} X / S=q$. Then for every irreducible component $D_{\alpha}$ of $D_{X / S}(\mathcal{E})_{\text {red }}$ such that $X_{\alpha}$ is reduced there exist 1) a finite number of irreducible components, $T, 1 \leqq i \leqq m$, of $D_{x / s}$ such that $Z_{i}=\left(Z_{X / S}\right)_{T_{i}}$ are reduced and irreducible, 2) for each $i$ a subvariety, $Y_{i}$, of $D_{X_{i} / T_{i}}\left(\mathcal{E}_{i}\right)_{\mathrm{red}}, X_{i}=X_{T_{i}}, \mathcal{E}_{i}=\mathcal{E}_{T_{i}}$, such that either $\operatorname{dim} \mathscr{R}_{i} / Y_{i}<q$ or $S\left(\mathscr{R}_{i}\right)=\widetilde{Z}_{i}$, with $\widetilde{Z}_{i}=\left(Z_{i}\right)_{Y_{i}}$ reduced, and $\mathscr{R}_{i}$ is torsion free as an $\mathcal{O}_{\tilde{\mathbb{Z}}_{i}}$-module, where $\left.\mathscr{R}_{i}=\left(\mathscr{R}_{X_{i} / T_{i}}\left(\mathcal{E}_{i}\right)\right)_{Y_{i}}, 3\right)$ an analytic subset, $N$, of $Y=Y_{1} \times{ }_{s} \cdots \times_{s} Y_{m}$, and finally 4) a weakly defined generically surjective meromorphic map $\bar{h}: N \rightarrow D_{a}$.

Proof. Let $u_{\alpha}: \mathcal{E}_{\alpha} \rightarrow \mathscr{R}_{\alpha}$ be the universal quotient restricted to $D_{\alpha}$. Let $\mathscr{R}_{\alpha}^{\prime}$ be the kernel of $u_{\alpha}$. Take an irredundant primary decomposition, $\mathscr{R}_{\alpha}^{\prime}=\bigcap_{i \in I} Q_{i}$, of $\mathscr{R}_{\alpha}^{\prime}$ in $\mathcal{E}_{\alpha}$ [22]. Let $\mathscr{F}_{i}=\mathcal{E}_{\alpha} / Q_{i}$ and $V_{i}$ the subvariety of $X_{\alpha}$ corresponding to $Q_{i}$. Then by Frisch [6] there exists a Zariski open subset $U$ of $D_{\alpha}$ such that $\mathscr{F}_{i}$ and $\mathcal{O}_{V_{i}}$ are flat over $U$ for all $i$. Note that since $f$ is proper, we may assume that $V_{i} \cap f^{-1}(U) \neq \varnothing$ for only finitely many $i$, say, for $i=1, \cdots, m$. On the other hand, since $V_{i}$ are flat over $U$, by the universality of $D_{X / S}$ there exists a unique $S$-morphism $\varphi_{i}: U \rightarrow D_{X / S}$ such that $Z_{U}=\left(V_{i}\right)_{U}$. Let $T_{i}$ be any irreducible component of $D_{X / S}$ which contains $\varphi_{i}(U)$. Then the reducedness and irreducibility of $Z_{i}$ follows from those of $Z_{U}=\left(V_{i}\right)_{U}$ and the flatness of $Z_{i}$ over $T_{i}$ as in the proof of the previous lemma. Since $\left(\mathscr{F}_{i}\right)_{U}$ is the quotient of $\left(\mathcal{E}_{i}\right)_{U} \cong\left(\mathcal{E}_{\alpha}\right)_{U}$ and flat over $U$, by the universality of $D_{X_{i} / T_{i}}\left(\mathcal{E}_{i}\right)$ we have 
a unique $T_{i}$-morphism $\tau_{i}: U \rightarrow D_{X_{i} / T_{i}}\left(\mathcal{E}_{i}\right)$ such that $\left(\mathscr{F}_{i}\right)_{U} \cong\left(\mathscr{R}^{i}\right)_{U}$, where $\mathscr{R}^{i}=\mathscr{R}_{X_{i} / T_{i}}\left(\mathcal{E}_{i}\right)$. Let $Y_{i}$ be a minimal analytic subset of $D_{X_{i} / T_{i}}\left(\mathcal{E}_{i}\right)_{\text {red }}$ containing $\tau_{i}(U)$ and $\mathscr{R}_{i}=\left(\mathscr{R}^{i}\right)_{Y_{i}}$.

Let $T=T_{1} \times{ }_{s} \cdots \times{ }_{s} T_{m}, \varphi=\varphi_{1} \times{ }_{s} \cdots \times{ }_{s} \varphi_{m}: U \rightarrow T$, and $Y=Y_{1} \times{ }_{s} \cdots \times{ }_{s} Y_{m}$. Define a $T$-morphism $\tau: U \rightarrow Y$ by $\tau=\tau_{1} \times{ }_{s} \cdots \times{ }_{s} \tau_{m}$, where $U$ is over $T$ by $\varphi$. Now let $\widetilde{\mathcal{R}}_{i}$ be the pull-backs on $X_{Y}$ of $\mathscr{R}_{i}$ by the natural maps $X_{Y} \rightarrow X_{Y_{i}}$. Then we have the natural quotient maps $q_{i}: \mathcal{E}_{Y} \rightarrow \widetilde{\mathcal{R}}_{i}$. Let $\widetilde{Q}_{i}$ be the kernel of $q_{i}$ and $\mathscr{F}=\mathcal{E}_{Y} / \bigcap_{i} \widetilde{Q}_{i}$. Let $N$ be the minimal analytic subset of $Y$ which contains $\tau(U)$. Let $W$ be the nonempty Zariski open subset of $N$ such that $\mathscr{F}_{N}$ is flat over $W$. Then by the universality of $D_{X / S}(\mathcal{E})$ we have a unique $S$-morphism $h: W \rightarrow D_{X / S}(\mathcal{E})$ such that $\mathscr{I}_{W}$ $\cong \mathscr{R}_{W}, \mathscr{R}=\mathscr{R}_{X / S}(\mathcal{E})$. Then by our construction we infer readily that $h \tau$ is the identity on $\tau^{-1}(W)$, which is not empty by our choice of $N$. In particular we have $h(W) \subseteq D_{\alpha}$ and it is dense there. Finally by Lemma $5.1 h$ defines a weakly defined surjective $S$-meromorphic map $\bar{h}: N$ $\rightarrow D_{\alpha}$.

We now show the property of $\mathcal{R}_{i}$ stated in 2) of the lemma. Since $\operatorname{dim} X / S \leqq q$, it is clear that $\operatorname{dim} \mathscr{R}_{i} / Y_{i} \leqq q$. Suppose that $\operatorname{dim} \mathscr{R}_{i} / Y_{i}=q$ for some $i$. Then since $\left(\mathscr{F}_{i}\right)_{U} \cong\left(\mathscr{R}^{i}\right)_{U}=\left(\mathscr{R}_{i}\right)_{U}$, we also have that $\operatorname{dim} \mathscr{F}_{i} / D_{\alpha}$ $=q$. Then $X_{\alpha}$ being reduced, supp $\mathscr{F}_{i}=V_{i}$ coincides with an irreducible component of $X_{\alpha}$. Since $\mathscr{F}_{i}$ is primary, $\mathscr{H}_{i}$ then turns out to be a torsion free $\mathcal{O}_{V_{i}}$-module. Therefore noting that $\mathscr{F}_{i}$ is flat over $U$, by 3 ) $\rightarrow 2$ ) of Lemma 5. 6 there is a dense Zariski open subset $U_{0}$ of $U$ such that $\mathscr{F}_{i, u}$ is torsion free as an $\mathcal{O}_{V_{i}, u}$-module for every $u \in U_{0}$. Hence in virtue of the relations $\left(\mathscr{F}_{i}\right)_{U} \cong\left(\mathscr{R}_{i}\right)_{U}$ and $Z_{U}=\left(\widetilde{Z}_{i}\right)_{U}=\left(V_{i}\right)_{U}, \mathscr{R}_{i, \tau(u)}, u \in U_{0}$, is a torsion free $\mathcal{O}_{\widetilde{Z}_{i}, \tau_{i}(u)}$-module, and $\widetilde{Z}_{i}$ is reduced, it being flat over $T_{i}$ 。 Also $\widetilde{X}_{i}=\left(X_{i}\right)_{Y_{i}}$ is reduced since it is flat over $Y_{i}$ and $\left(\widetilde{X}_{i}\right)_{U}=X_{U}$ is reduced, where $U$ is over $Y_{i}$ by $\tau_{i}$. Moreover since $q=\operatorname{dim} V_{i} / D_{\alpha}=\operatorname{dim} \widetilde{Z}_{i}$ $/ Y_{i}=\operatorname{dim} \widetilde{X}_{i} / Y_{i}, \widetilde{Z}_{i}$ is an irreducible component of $\widetilde{X}_{i}$. From these facts and the minimality of $Y_{i}$ it follows that $\operatorname{supp} \mathscr{R}_{i}=\widetilde{Z}_{i}$ and hence $S\left(\mathcal{R}_{i}\right)=\widetilde{Z}_{i}$ since $\widetilde{X}_{i}$ is reduced. Finally torsion freeness of $\mathscr{R}_{i}$ as an $\mathcal{O}_{\widetilde{Z}_{i}}$-module follows from Lemma 5.6 2) $\rightarrow 3$ ).

Q.E.D.

Proof of Theorem 5.2. Let $D_{\alpha}$ be any irreducible component of $D_{X / S}(\mathcal{E})_{\text {red. }}$ Then we prove the theorem by induction on $q=\operatorname{dim} \mathscr{R}_{\alpha} / D_{\alpha}$. 
Firstly if $q=-1$, then $\mathscr{R}_{\alpha}=\{0\}$ so that $D_{a} \cong S$ and hence proper over $S$. So assume that $q \geqq 0$. Then by Lemma 5.8 it suffices to show the properness of $B_{0} \times{ }_{T} \cdots \times{ }_{T} B_{n}$ in the notation of that lemma. This in turn follows from the properness of $T$ over $S$ and $B_{k}$ over $T$ for all $k$. Since $Z_{T}$ is reduced by 1 ) of the lemma, the properness of $T$ follows from Lemma 3.5, Proposition 3. 4 and Remark 3.5. We shall show that $B_{k}$ are proper over $T$. First by 1 ) and by the definition of $B_{k}$ in 3 ) of that lemma, taking $X=Z_{T}, S=T, \mathcal{E}=\mathcal{E}_{k}$ and $D_{\alpha}=B_{k}$ we can reduce the problem to the case where $f$ is flat, $X_{\alpha}$ is reduced and $\operatorname{dim} X / S=q$. (Note that $Z_{T} \rightarrow T$ is a Kähler morphism.) Then we can apply Lemma 5. 9 to see that it is enough to show the properness of $N$ over $S$ in the notation of that lemma. $B_{y} 3$ ) and the definition of $Y$ in that lemma this follows from the properness of $Y_{i}$ over $T_{i}$ and $T_{i}$ over $S$ in the notations there. Since $Z_{i}$ are reduced, by Proposition 3.4 $T_{i}$ are proper over $S . \quad$ As for $Y_{i}$ if $\operatorname{dim} \mathscr{R}_{i} / Y_{i}<q$, then noting that $X_{i} \rightarrow T_{i}$ is a Kähler morphism, and applying the induction hypothesis to an irreducible component $Y_{i}^{\prime}$ of $D_{X_{i} / T_{i}}\left(\mathcal{E}_{i}\right)_{\text {red }}$ which contains $Y_{i}$ (we still have that $\operatorname{dim} \mathscr{R}_{Y_{i}^{\prime}} / Y_{i}^{\prime}$ $<q, \mathscr{R}_{Y_{i}^{\prime}}=\left(\mathscr{R}_{X_{i} / T_{i}}\left(\mathcal{E}_{i}\right)\right) Y_{i}^{\prime}$, by the flatness of $\left.\mathscr{R}_{Y_{i}^{\prime}}\right)$, we get that $Y_{i}^{\prime}$ and hence $Y_{i}$ is proper over $T_{i}$. So suppose that $\operatorname{dim} \mathscr{R}_{i} / Y_{i}=q$. In this case $S\left(\mathscr{R}_{i}\right)=\widetilde{Z}_{i}$ and $\mathcal{R}_{i}$ is torsion free as an $\mathcal{O}_{\widetilde{Z}_{i}}$ module by 2 ) of that lemma. Hence we can consider $Y_{i}$ as a subspace of $D_{Z_{i}, T_{i}}\left(\bar{\varepsilon}_{i}\right)$, where $\bar{\varepsilon}_{i}=\varepsilon_{i}$ $\otimes \mathcal{O X}_{i} \mathcal{O}_{Z_{i}}$. Thus taking $X=Z_{i}, S=T_{i}, D_{\alpha}=$ an irreducible component of $D_{Z_{i} / T_{i}}\left(\varepsilon_{i}\right)$ containing $Y_{i}$, and $\varepsilon=\overline{\mathcal{E}}_{i}$, we can reduce the problem to the case where $f: X \rightarrow S$ is flat, $\operatorname{dim} X / S=q, X$ and $S$ are reduced and irreducible, $X_{\alpha}$ is reduced, and $\mathscr{R}_{\alpha}$ is torsion free on $X_{a}$. (In fact use Lemma 1.4 (Lemma 5.6) to get the reducedness of $X_{a}$ (torsion freeness of $\mathcal{R}_{\alpha}$ ) from that of $\widetilde{Z}_{i}\left(\mathscr{R}_{i}\right)$.) Then we can apply Lemma 5.7 and the properness of $D_{\alpha}$ follow's immediately from that lemma.

Q.E.D.

Finally the proof of Theorem 5. 3 is quite analogous and so we only indicate the necessary modifications. First in view of the above proof, especially Lemmas 5.8 and 5.9, we have to consider also the relative form of the class $\mathscr{C}$, which we shall define as follows. Let $f: X \rightarrow S$ be a proper morphism of complex spaces with $X$ reduced. Then we say that $f$ belongs to the class $\mathscr{C} / S$, denoted by $f \in \mathscr{C} / S$, if for every point 
$s \in S$ there is a neighborhood $U$ of $s$ in $S$ and a proper Kähler morphism $h: Y \rightarrow U$ with a surjective $U$-meromorphic map $\phi: Y \rightarrow X$. Then we actually prove the theorem in the following form; let $f: X \rightarrow S$ be a proper morphism with $f \in \mathscr{C} / S$ and $\mathcal{E}$ a coherent analytic sheaf on $X$. Then every irreducible component $D_{a}$ of $D_{X / S}(\varepsilon)_{\text {red }}$ is proper orer $S$.

For this purpose first one formulate and prove the relative form of Lemma 4.6 almost as in the absolute case. From this it follows that $X$ has the property $B P$ and bence also $\bar{D} P$ almost as in the proof of Theorem 4.9. Finally one sees easily that the last assertion of Lemma 5. 7 is then still true even if we assume that $f \in \mathscr{C} / S$ instead of being a Kähler morphism. Then the rest of the proof is quite the same as above.

\section{References}

[1] Artin, M., Algebraization of formal moduli, I, II, in Collected Papers in Honor of K. Kodaira, Univ. of Tokyo Press and Princeton Univ. Press. (1969), 21-72, and Ann. of Math., 91 (1970), 88-135.

[2] Banica, C., Un théorème concernant les familles analytiques d'espace complexes, Rev. Roumaine Math. Pures Appl., 18 (1973), 1515-1520.

[3] Barlet, D., Espace analytique réduit des cycles onalytiques complexes de dimension finie, Seminaire F. Norguet, Lecture Notes in Nath., 482 Springer (1975), 1-158.

[4] Bishop, E., Conditions for the analyticity of certain set", Michigan Math. J., 11 (1964), 289-304.

[5] Douady, A., Le Problème de modules pour ler sous-espaces analytiques complexes d'un espace analytique donné, Ann. Inst. Fourier. Grenoble, 16 (1966), 1-95.

[6] Frisch, J., Points de platitude d'un morphisme d'espaces analytiques complexes, Invent. Math., 4 (1967), 118-138.

[7] Fujiki, A., On the blowing down of analytic spaces, Publ. RIMS, Kyolo Univ., 10 (1975), 473-507.

[8] Fujiki, A., On automorphism groups of compact Kähler manifolds, to appear.

[9] Fujiki, A., Countability of the Douady space of a complex space, io appear.

[10] Grothendieck, A., Technique de construction en géométrie analytique, Séminaire Henri Cartan, 13 année (1960/61).

[11] Gunning, R. C. and Rossi, H., Analytic functions of several complex variables. Prentice Hall, Englewood Cliffs, N. J. (1965).

[12] Harvey, R. and Shiffman, B., A characterization of holomorphic chains, Ann. of Math., 99 (1974), 553-587.

[13] Herrera, M., Integration on a semianalytic set. Bull. Soc. Math. France, 94 (1966), 141-180.

[14] Hironaka, H., Flattening theorem in complex-analytic geometry, Amer. J. Math., 97 (1975), 503-547.

[15] King, J., The currents defined by analytic varieties, Acta Math., 127 (1971), 185-2:20.

[16] Moisezon. B. G., Singular Kählerian spaces, Proc. Int. Conf. on Manifolds and related topics in topology, Univ. of Tokyo Press (1974), 343-351.

[17] Pourcin, G., Théorème de Douady audessus de S, Ann. Sci. Norm. Sup. di Pisa., 
XXIII, III (1969), 451-459.

[18] Rossi, H., Picard varieties of an isolated singular point, Rice Univ. Studies, 54 (1968), 63-73.

[19] Šafarevič, I. R. et al., Algebraic surfaces, Steklov Institut of Math. 75, English translation, Providence, Rhode Island, Amer. Math. Soc., (1967).

[20] Shiffman, B., Extending analytic varieties, in symposium on several complex variables, Lecture Notes in Math., 184 (1971), 208-222.

[21] Siu, Y.-T., Extension of meromorphic maps into Kähler manifolds, Ann. of Math., 102 (1975), 421-462.

[22] Siu, Y.-T., Noether-Lasker decomposition of coherent analytic subsheaves, Trans. Amer. Math. Soc., 135 (1969), 375-385.

[23] Stoll, W., The continuity of the fiber integral, Math. Z., 95 (1967), 87-138.

[24] Lieberman, D. I., Compactness of the Chow scheme: Applications to automorphisms and deformations of Kähler manifolds, preprint. 